\title{
Radiative double copy for Einstein-Yang-Mills theory
}

\author{
David Chester* \\ Mani Bhaumik Institute of Theoretical Physics, Department of Physics and Astronomy, UCLA, \\ Los Angeles, California 90095-1547, USA
}

(Received 27 December 2017; published 17 April 2018)

\begin{abstract}
Recently, a double-copy formalism was used to calculate gravitational radiation from classical YangMills radiation solutions. This work shows that the Yang-Mills theory coupled to a biadjoint scalar field admits a radiative double copy that agrees with solutions in the Einstein-Yang-Mills theory at the lowest finite order. Within this context, the trace-reversed metric $\bar{h}^{\mu \nu}$ is a natural double copy of the gauge boson $A^{\mu a}$. This work provides additional evidence that solutions in gauge and gravity theories are related, even though their respective Lagrangians and nonlinear equations of motion appear to be different.
\end{abstract}

DOI: 10.1103/PhysRevD.97.084025

\section{INTRODUCTION}

The Lagrangians and equations of motion for gauge and gravity theories appear to be rather different. Nevertheless, there are intriguing double-copy connections between their solutions. This includes the Kawai-Lewellen-Tye (KLT) tree-level relations between gauge and gravity amplitudes in string theory [1] and the Bern-Carrasco-Johansson (BCJ) double-copy relations between diagrams in quantum field theory [2]. The BCJ double-copy relations are based on color-kinematics duality, which gives particularly simple constructions of gravity amplitudes starting from gaugetheory amplitudes.

At tree level the BCJ amplitude relations are proven [3-7]. Numerous calculations at higher loops provide evidence for the loop-level double-copy conjecture [8-11], and progress has been made to understand analogous monodromy relations, extending KLT relations to loop level [12-17]. Einstein-Yang-Mills scattering amplitudes [18-21] can also be found via the double copy [22-24] using the Cachazo-HeYuan (CHY) formalism [25]. Biadjoint scalar fields can be used to find solutions in Yang-Mills [26], and solutions in a Yang-Mills-biadjoint-scalar theory have been shown to give scattering amplitudes in Einstein-Yang-Mills [27-29].

With the recent experimental detection of gravitational waves by LIGO [30], precision calculational tools for gravitational wave emission are essential. Exploiting color-kinematics duality to relate radiation solutions between Yang-Mills and general relativity is attractive

\footnotetext{
*dchester@ucla.edu
}

Published by the American Physical Society under the terms of the Creative Commons Attribution 4.0 International license. Further distribution of this work must maintain attribution to the author(s) and the published article's title, journal citation, and DOI. Funded by SCOAP. because general relativity is difficult to solve and the double copy has been shown to work for a wide variety of gravity theories [31-33] The connection between radiation solutions of gauge theory and gravity has been described recently [34-40]. The first example of using the radiative double copy to find nonlinear terms in general relativity utilized perturbative Yang-Mills solutions [41]. Similarly a biadjoint scalar field can be used to find YangMills radiation [42].

This work builds off the radiative double copy for general relativity found by Goldberger and Ridgway [41] to find gravitational radiation in Einstein-Yang-Mills theory. By comparing the differential equations of the sources and fields in gauge theory and gravity, radiative diagrams are used to represent specific algebraic terms. Solutions in gravity can be found from Yang-Mills theory, and the diagrams with threepoint vertices can be computed by stitching lower-order solutions together. At leading order, the trace-reversed metric [43], $\bar{h}^{\mu \nu}$, is a natural double copy of the Yang-Mills potential $A^{\mu a}$ [44]. Motivation for a perturbative double copy can be seen at the Lagrangian level, as the linearized gravity Lagrangian is quite similar to the QED Lagrangian, a linearized version of the Yang-Mills Lagrangian. Similarly, these two theories both have an analogous linearized wave equation. Remarkably, radiation solutions of nonlinear gauge and gravity theories are related, at least when iterated perturbatively. A double copy of Yang-Millsadjoint-scalar theory is also briefly mentioned, which can recover radiation solutions in the Einstein-Maxwell theory.

While this paper focuses on classical solutions that could be calculated with more traditional methods [45-54], the hope is that the radiative double copy could help with difficult calculations that may be more cumbersome to do in general relativity alone. As more experimental data for gravitational radiation is collected, new methods for calculating complicated radiation processes are encouraged. 
Section II calculates radiation in the Yang-Mills-biadjoint-scalar theory. Section III calculates radiation in the Einstein-Yang-Mills theory and the double copy is confirmed by direct calculation. Section IV states our concluding remarks. Appendix A calculates details of the gravitational contribution to the energy momentum pseudotensor and Appendix B gives radiative Feynman rules for simple diagrams with three-point vertices.

\section{RADIATION IN YANG-MILLS- BIADJOINT-SCALAR THEORY}

\section{A. Equations of motion and initial conditions}

In this section, the non-Abelian radiation field for the Yang-Mills-biadjoint-scalar field theory is computed to first order in the weak-field approximation. To start, the Lagrangian associated with the Yang-Mills-biadjoint-scalar theory is

$$
\begin{aligned}
\mathcal{L}= & -\frac{1}{4} F_{\mu \nu}^{a} F^{\mu \nu a}+\frac{1}{2} D_{\mu} \Phi^{\tilde{a} a} D^{\mu} \Phi^{\tilde{a} a}-\frac{y}{3} f^{a b c} f^{\tilde{a} \tilde{b} \tilde{c}} \Phi^{\tilde{a} a} \Phi^{\tilde{b} b} \Phi^{\tilde{c} c} \\
& -g J^{\mu a} A_{\mu}^{a}-y J^{\tilde{a} a} A^{\tilde{a} a}
\end{aligned}
$$

where $f^{a b c}$ and $f^{\tilde{a} \tilde{b} \tilde{c}}$ refer to structure constants of different groups, the biadjoint scalar $\Phi^{\tilde{a} a}$ has an index associated with each gauge group, and $y=-i g \tilde{g} / 2$ relates the conventions of Ref. [27] with the conventions of Refs. [26,42]. In principle, there could be an $\mathcal{O}\left(\Phi^{4}\right)$ term in the Lagrangian, but the coupling constant would have different dimensions than $y$ and is not needed for the double copy. The non-Abelian field strength is given by

$F_{\mu \nu}^{a}(x)=\partial_{\mu} A_{\nu}^{a}(x)-\partial_{\nu} A_{\mu}^{a}(x)-g f^{a b c} A_{\mu}^{b}(x) A_{\nu}^{c}(x)$,

and the mostly minus metric will be used, such that $\eta^{\mu \nu}=\operatorname{diag}(1,-1,-1,-1)$. The covariant derivative is given by

$$
D_{\mu} \Phi^{\tilde{a} a}(x)=\partial_{\mu} \Phi^{\tilde{a} a}(x)-g f^{a b c} A_{\mu}^{b}(x) \Phi^{\tilde{a} c}(x) .
$$

The equations of motion for the Yang-Mills field is

$$
D_{\mu} F^{\mu \nu a}(x)-g f^{a b c} \Phi^{\tilde{a} b}(x) D^{\nu} \Phi^{\tilde{a} c}(x)=g J^{\nu a}(x),
$$

where $J^{\mu a}(x)$ is a non-Abelian vector current acting as a source for the Yang-Mills field and is covariantly conserved, such that $D_{\mu} J^{\mu a}=0$. The equation of motion for the biadjoint scalar field is

$$
\begin{aligned}
& \partial_{\mu} D^{\mu} \Phi^{\tilde{a} a}(x)-g f^{a b c} A_{\mu}^{b}(x) D^{\mu} \Phi^{\tilde{a} c}(x) \\
& \quad-y f^{a b c} f^{\tilde{a} \tilde{b} \tilde{c}} \Phi^{\tilde{b} b}(x) \Phi^{\tilde{c} c}(x)=y J^{\tilde{a} a}(x) .
\end{aligned}
$$

For $N$ colliding charged particles, the worldline of particle $\alpha$ is $x_{\alpha}^{\mu}(\tau)=b_{\alpha}^{\mu}+v_{\alpha}^{\mu} \tau$ for $\tau \rightarrow-\infty$. These initial conditions specify an impact parameter $b_{\alpha \beta}^{\mu}=b_{\alpha}^{\mu}-b_{\beta}^{\mu}$ and a constant initial velocity $v_{\alpha}^{\mu}$ which satisfies $v_{\alpha}^{2}=1$. For arbitrary times near and after the collision,

$$
x_{\alpha}^{\mu}(\tau)=b_{\alpha}^{\mu}+v_{\alpha}^{\mu} \tau+z_{\alpha}^{\mu}(\tau),
$$

where $z_{\alpha}^{\mu}(\tau)$ is the deflection due to the Yang-Mills and biadjoint scalar fields. The vector source for $N$ colliding charged particles is

$$
J^{\mu a}(x)=\sum_{\alpha=1}^{N} \int d \tau c_{\alpha}^{a}(\tau) v_{\alpha}^{\mu}(\tau) \delta^{d}\left(x-x_{\alpha}(\tau)\right),
$$

where $\alpha$ is a particle number label, $v_{\alpha}^{\mu}(\tau)=\frac{d \alpha_{\alpha}^{\mu}(\tau)}{d \tau}$ is the velocity, and $c_{\alpha}^{a}(\tau)$ is the associated adjoint color charge [55]. The biadjoint source $J^{\tilde{a} a}(x)$ for $N$ particles is

$$
J^{\tilde{a} a}(x)=\sum_{\alpha=1}^{N} \int d \tau c_{\alpha}^{\tilde{a}}(\tau) c_{\alpha}^{a}(\tau) \delta^{d}\left(x-x_{\alpha}(\tau)\right),
$$

where it is assumed that the color charges $c_{\alpha}^{\tilde{a}}(\tau)$ and $c_{\alpha}^{a}(\tau)$ are in two different gauge groups.

The Lorenz gauge is taken by setting $\partial_{\mu} A^{\mu a}=0$. In order to simplify these equations, the explicit dependence on the covariant derivatives is removed and gauge dependent sources $\hat{J}^{\mu a}$ and $\tilde{J}^{\tilde{a} a}$ are defined such that

$$
\square A^{\mu a}(x)=g \hat{J}^{\mu a}(x), \quad \square \Phi^{\tilde{a} a}=y \hat{J}^{\tilde{a} a},
$$

where $\square \equiv \partial_{\nu} \partial^{\nu}$. With these definitions, the pseudovector source is

$\hat{J}^{\mu a}=J^{\mu a}+f^{a b c}\left[A_{\nu}^{b}\left(\partial^{\nu} A^{\mu c}+F^{\nu \mu c}\right)+\Phi^{\tilde{a} b} D^{\mu} \Phi^{\tilde{a} c}\right]$,

where the pseudovector is locally conserved, $\partial_{\mu} \hat{J}^{\mu a}=0$. The pseudoscalar source is given by

$$
\begin{aligned}
\hat{J}^{\tilde{a} a}= & J^{\tilde{a} a}+\frac{g}{y} f^{a b c}\left[\partial_{\mu}\left(A^{\mu b} \Phi^{\tilde{a} c}\right)+A^{\mu b} D_{\mu} \Phi^{\tilde{a} c}\right. \\
& \left.+\frac{y}{g} f^{\tilde{a} \tilde{b} \tilde{c}} \Phi^{\tilde{b} b} \Phi^{\tilde{c} c}\right] .
\end{aligned}
$$

Similar to the worldline $x_{\alpha}^{\mu}(\tau)$, the color charges are dynamical and are given initial conditions $c_{\alpha}^{a}(\tau)=c_{\alpha}^{a}$ and $c_{\alpha}^{\tilde{a}}(\tau)=c_{\alpha}^{\tilde{a}}$ for $\tau \rightarrow-\infty$. For times near and after the collision,

$$
c_{\alpha}^{a}(\tau)=c_{\alpha}^{a}+\bar{c}_{\alpha}^{a}(\tau), \quad c_{\alpha}^{\tilde{a}}(\tau)=c_{\alpha}^{\tilde{a}}+\bar{c}_{\alpha}^{\tilde{a}}(\tau),
$$

where $\bar{c}_{\alpha}^{a}(\tau)$ and $\bar{c}_{\alpha}^{\tilde{a}}(\tau)$ are the corrections due to the YangMills and biadjoint scalar fields. The time evolution of the momentum is 


$$
\begin{aligned}
\frac{d p_{\alpha}^{\mu}(\tau)}{d \tau}= & g c_{\alpha}^{a}(\tau) F^{\mu \nu a}\left(x_{\alpha}(\tau)\right) v_{\alpha \nu}(\tau) \\
& -y \partial^{\mu} \Phi^{\tilde{a} a}\left(x_{\alpha}(\tau)\right) c_{\alpha}^{a}(\tau) c_{\alpha}^{\tilde{a}}(\tau),
\end{aligned}
$$

and the time evolution of the charges is

$$
\begin{aligned}
\frac{d c_{\alpha}^{a}(\tau)}{d \tau}= & g f^{a b c} v_{\alpha}^{\mu}(\tau) A_{\mu}^{b}\left(x_{\alpha}(\tau)\right) c_{\alpha}^{c}(\tau) \\
& -y f^{a b c} \Phi^{\tilde{b} b}\left(x_{\alpha}(\tau)\right) c_{\alpha}^{\tilde{b}}(\tau) c_{\alpha}^{c}(\tau), \\
\frac{d c_{\alpha}^{\tilde{a}}(\tau)}{d \tau}= & -y f^{\tilde{a} \tilde{b} \tilde{c}} \Phi^{\tilde{b} b}\left(x_{\alpha}(\tau)\right) c_{\alpha}^{b}(\tau) c_{\alpha}^{\tilde{c}}(\tau) .
\end{aligned}
$$

These summarize all of the equations needed to iteratively solve for radiation in Yang-Mills-biadjoint-scalar theory within a weak-field approximation.

\section{B. Solutions of the radiation fields}

For weak fields, the lowest-order sources can be found from the initial conditions. The pseudocurrents in momentum space are

$$
\begin{aligned}
& \left.\hat{J}^{\mu a}(k)\right|_{\mathcal{O}\left(g^{0}\right)}=\sum_{\alpha=1}^{N} e^{i k \cdot b_{\alpha}}(2 \pi) \delta\left(k \cdot v_{\alpha}\right) v_{\alpha}^{a} c_{\alpha}^{a}, \\
& \left.\hat{J}^{\tilde{a} a}(k)\right|_{\mathcal{O}\left(y^{0}\right)}=\sum_{\alpha=1}^{N} e^{i k \cdot b_{\alpha}}(2 \pi) \delta\left(k \cdot v_{\alpha}\right) c_{\alpha}^{\tilde{a}} c_{\alpha}^{a},
\end{aligned}
$$

which can be utilized to find the Yang-Mills and biadjoint scalar fields to lowest order from Eq. (2.9), giving

$$
\begin{aligned}
& \left.A^{\mu a}(x)\right|_{\mathcal{O}\left(g^{1}\right)}=-g \sum_{\alpha=1}^{N} \int_{l}(2 \pi) \delta\left(l \cdot v_{\alpha}\right) \frac{e^{-i l \cdot\left(x-b_{\alpha}\right)}}{l^{2}} v_{\alpha}^{\mu} c_{\alpha}^{a}, \\
& \left.\Phi^{\tilde{a} a}(x)\right|_{\mathcal{O}\left(y^{1}\right)}=-y \sum_{\alpha=1}^{N} \int_{l}(2 \pi) \delta\left(l \cdot v_{\alpha}\right) \frac{e^{-i l \cdot\left(x-b_{\alpha}\right)}}{l^{2}} c_{\alpha}^{\tilde{a}} c_{\alpha}^{a} .
\end{aligned}
$$

The lowest-order fields can be used to find the deflections of the sources, given by

$$
\begin{aligned}
\left.m_{\alpha} \frac{d^{2} z_{\alpha}^{\mu}(\tau)}{d \tau^{2}}\right|_{\mathcal{O}\left(g^{2}\right)}= & g c_{\alpha}^{a}\left(\left.\partial^{\mu} A^{\nu a}\left(x_{\alpha}(\tau)\right)\right|_{\mathcal{O}\left(g^{1}\right)}\right. \\
& \left.-\left.\partial^{\nu} A^{\mu a}\left(x_{\alpha}(\tau)\right)\right|_{\mathcal{O}\left(g^{1}\right)}\right) v_{\alpha \nu}, \\
\left.m_{\alpha} \frac{d^{2} z_{\alpha}^{\mu}(\tau)}{d \tau^{2}}\right|_{\mathcal{O}\left(y^{2}\right)}= & -\left.y \partial^{\mu} \Phi^{\tilde{a} a}\left(x_{\alpha}(\tau)\right)\right|_{\mathcal{O}\left(y^{1}\right)} c_{\alpha}^{\tilde{a}} c_{\alpha}^{a} .
\end{aligned}
$$

Plugging in the derivatives of the lowest-order fields gives

$$
\begin{aligned}
\left.m_{\alpha} \frac{d^{2} z_{\alpha}^{\mu}(\tau)}{d \tau^{2}}\right|_{\mathcal{O}\left(g^{2}\right)}= & i g^{2} \sum_{\beta \neq \alpha}\left(c_{\alpha}^{a} c_{\beta}^{a}\right) \int_{l}(2 \pi) \delta\left(l \cdot v_{\beta}\right) \frac{\left.e^{-i l \cdot\left(b_{\alpha \beta}+v_{\alpha} \tau\right.}\right)}{l^{2}} \\
& \times\left[\left(v_{\alpha} \cdot v_{\beta}\right) l^{\mu}-\left(v_{\alpha} \cdot l\right) v_{\beta}^{\mu}\right] \\
\left.m_{\alpha} \frac{d^{2} z_{\alpha}^{\mu}(\tau)}{d \tau^{2}}\right|_{\mathcal{O}\left(y^{2}\right)}= & -i y^{2} \sum_{\beta \neq \alpha}\left(c_{\alpha}^{a} c_{\beta}^{a}\right) c_{\alpha}^{\tilde{a}} c_{\beta}^{\tilde{a}} \int_{l}(2 \pi) \delta\left(l \cdot v_{\beta}\right) \\
& \times \frac{\left.e^{-i l \cdot\left(b_{\alpha \beta}+v_{\alpha} \tau\right.}\right)}{l^{2}} l^{\mu} .
\end{aligned}
$$

Note that writing the color charge contraction as $c_{\alpha} \cdot c_{\beta}$ would be ambiguous with our notation, as $c_{\alpha}^{a} c_{\beta}^{a}$ and $c_{\alpha}^{\tilde{a}} c_{\beta}^{\tilde{a}}$ are distinct. The first correction of the color charges to second order in $g$ is given by

$$
\begin{aligned}
& \left.\frac{d \bar{c}_{\alpha}^{a}(\tau)}{d \tau}\right|_{\mathcal{O}\left(g^{2}\right)}=\left.g f^{a b c} v_{\alpha}^{\mu} A_{\mu}^{b}\left(x_{\alpha}(\tau)\right)\right|_{\mathcal{O}\left(g^{1}\right)} c_{\alpha}^{c}, \\
& \left.\frac{d \bar{c}_{\alpha}^{a}(\tau)}{d \tau}\right|_{\mathcal{O}\left(y^{2}\right)}=-\left.y f^{a b c} \Phi^{\tilde{b} b}\left(x_{\alpha}(\tau)\right)\right|_{\mathcal{O}\left(y^{1}\right)} c_{\alpha}^{\tilde{b}} c_{\alpha}^{c} \\
& \left.\frac{d \bar{c}_{\alpha}^{\tilde{a}}(\tau)}{d \tau}\right|_{\mathcal{O}\left(y^{2}\right)}=-\left.y f^{\tilde{a} \tilde{b} \tilde{c}} \Phi^{\tilde{b} b}\left(x_{\alpha}(\tau)\right)\right|_{\mathcal{O}\left(y^{1}\right)} c_{\alpha}^{b} c_{\alpha}^{\tilde{c}}
\end{aligned}
$$

Once again, plugging in the lowest-order fields gives

$$
\begin{aligned}
\left.\frac{d \bar{c}_{\alpha}^{a}(\tau)}{d \tau}\right|_{\mathcal{O}\left(g^{2}\right)}= & -g^{2} \sum_{\beta \neq \alpha} f^{a b c} c_{\beta}^{b} c_{\alpha}^{c}\left(v_{\alpha} \cdot v_{\beta}\right) \\
& \times \int_{l}(2 \pi) \delta\left(l \cdot v_{\beta}\right) \frac{e^{-i l \cdot\left(b_{\alpha \beta}+v_{\alpha} \tau\right)}}{l^{2}} \\
\left.\frac{d \bar{c}_{\alpha}^{a}(\tau)}{d \tau}\right|_{\mathcal{O}\left(y^{2}\right)}= & y^{2} \sum_{\beta \neq \alpha} f^{a b c} c_{\beta}^{b} c_{\alpha}^{c} c_{\alpha}^{\tilde{b}} c_{\beta}^{\tilde{b}} \int_{l}(2 \pi) \delta\left(l \cdot v_{\beta}\right) \\
& \times \frac{e^{-i l \cdot\left(b_{\alpha \beta}+v_{\alpha} \tau\right)}}{l^{2}}, \\
\left.\frac{d \bar{c}_{\alpha}^{\tilde{a}}(\tau)}{d \tau}\right|_{\mathcal{O}\left(y^{2}\right)}= & y^{2} \sum_{\beta \neq \alpha} f^{\tilde{a} \tilde{b} \tilde{c}} c_{\beta}^{\tilde{b}} c_{\alpha}^{\tilde{c}} c_{\alpha}^{b} c_{\beta}^{b} \int_{l}(2 \pi) \delta\left(l \cdot v_{\beta}\right) \\
& \times \frac{e^{-i l \cdot\left(b_{\alpha \beta}+v_{\alpha} \tau\right)}}{l^{2}} .
\end{aligned}
$$

These deflections can be utilized to find the sources to next order, which give the lowest-order radiation fields.

Taking the Fourier transform of Eq. (2.7) and integrating over the delta function gives

$$
J^{\mu a}(k)=\sum_{\alpha=1}^{N} \int d \tau e^{i k \cdot x_{\alpha}(\tau)}\left(v_{\alpha}^{\mu}+\frac{d z_{\alpha}^{\mu}(\tau)}{d \tau}\right)\left(c_{\alpha}^{a}+\bar{c}_{\alpha}^{a}(\tau)\right) .
$$

Expanding these results perturbatively in $g$ and $y$ gives 


$$
J^{\mu a}(k)=\sum_{\alpha=1}^{N} \int d \tau e^{i k \cdot\left(b_{\alpha}+v_{\alpha} \tau\right)}\left(\left(1+i k \cdot z_{\alpha}\right) v_{\alpha}^{\mu} c_{\alpha}^{a}+v_{\alpha}^{\mu} \bar{c}_{\alpha}^{a}+\frac{d z_{\alpha}^{\mu}}{d \tau} c_{\alpha}^{a}\right)+\mathcal{O}\left(g^{2} y^{2}\right),
$$

where explicit $\tau$ dependence has been suppressed and only terms to second order in $g$ or $y$ are kept. Integrating Eqs. (2.18) and (2.20) allows for the second-order current to be found, which has Yang-Mills and biadjoint scalar contributions given by

$$
\begin{aligned}
& \left.J^{\mu a}(k)\right|_{\mathcal{O}\left(g^{2}\right)}=g^{2} \sum_{\substack{\alpha=1 \\
\beta \neq \alpha}}^{N} \int_{l_{\alpha}, l_{\beta}} \mu_{\alpha, \beta}(k) \frac{l_{\alpha}^{2}}{k \cdot v_{\alpha}}\left[i f^{a b c} c_{\alpha}^{b} c_{\beta}^{c}\left(v_{\alpha} \cdot v_{\beta}\right) v_{\alpha}^{\mu}+\frac{c_{\alpha}^{b} c_{\beta}^{b}}{m_{\alpha}} c_{\alpha}^{a}\left\{-v_{\alpha} \cdot v_{\beta}\left(l_{\beta}^{\mu}-\frac{k \cdot l_{\beta}}{k \cdot v_{\alpha}} v_{\alpha}^{\mu}\right)+k \cdot v_{\alpha} v_{\beta}^{\mu}-k \cdot v_{\beta} v_{\alpha}^{\mu}\right\}\right], \\
& \left.J^{\mu a}(k)\right|_{\mathcal{O}\left(y^{2}\right)}=y^{2} \sum_{\substack{\alpha=1 \\
\beta \neq \alpha}}^{N} c_{\alpha}^{\tilde{a}} c_{\beta}^{\tilde{a}} \int_{l_{\alpha}, l_{\beta}} \mu_{\alpha, \beta}(k) \frac{l_{\alpha}^{2}}{k \cdot v_{\alpha}}\left[\frac{c_{\alpha}^{b} c_{\beta}^{b}}{m_{\alpha}} c_{\alpha}^{a}\left(l_{\beta}^{\mu}-\frac{k \cdot l_{\beta}}{k \cdot v_{\alpha}} v_{\alpha}^{\mu}\right)-i f^{a b c} c_{\alpha}^{b} c_{\beta}^{c} v_{\alpha}^{\mu}\right],
\end{aligned}
$$

where an extra integral over $l_{\alpha}$ was added with a momentum conserving delta function such that $k=l_{\alpha}+l_{\beta}$ and

$$
\begin{aligned}
\mu_{\alpha, \beta}(k)= & {\left[(2 \pi) \delta\left(v_{\alpha} \cdot l_{\alpha}\right) \frac{e^{i l_{\alpha} \cdot b_{\alpha}}}{l_{\alpha}^{2}}\right]\left[(2 \pi) \delta\left(v_{\beta} \cdot l_{\beta}\right) \frac{e^{i l_{\beta} \cdot b_{\beta}}}{l_{\beta}^{2}}\right](2 \pi)^{d} } \\
& \times \delta^{d}\left(k-l_{\alpha}-l_{\beta}\right) .
\end{aligned}
$$

The nonlinear field contributions to the pseudovector are represented by $j^{\mu a}$, which gives the following second order contributions:

$$
\begin{aligned}
\left.j^{\mu a}(k)\right|_{\mathcal{O}\left(g^{2}\right)}= & g^{2} \sum_{\substack{\alpha=1 \\
\beta \neq \alpha}}^{N} i f^{a b c} c_{\alpha}^{b} c_{\beta}^{c} \int_{l_{\alpha}, l_{\beta}} \mu_{\alpha, \beta}(k) \\
& \times\left[2 k \cdot v_{\beta} v_{\alpha}^{\mu}-v_{\alpha} \cdot v_{\beta} l_{\alpha}^{\mu}\right], \\
\left.j^{\mu a}(k)\right|_{\mathcal{O}\left(y^{2}\right)}= & y^{2} \sum_{\substack{\alpha=1 \\
\beta \neq \alpha}}^{N} i f^{a b c} c_{\alpha}^{b} c_{\beta}^{c} c_{\alpha}^{\tilde{a}} c_{\beta}^{\tilde{a}} \int_{l_{\alpha}, l_{\beta}} \mu_{\alpha, \beta}(k) l_{\alpha}^{\mu} .
\end{aligned}
$$

While $J^{\mu a}$ and $j^{\mu a}$ were computed algebraically, they also can be represented diagrammatically. Figure 1 depicts the diagrams associated with $\hat{J}^{\mu a}$ to second order in $g$ and $y$. The six diagrams are defined as

$$
\begin{aligned}
(1 \mathrm{a})^{\mu a}(k) \equiv & \sum_{\alpha=1}^{N} \int d \tau e^{i k \cdot\left(b_{\alpha}+v_{\alpha} \tau\right)} \\
& \times\left(\left.v_{\alpha}^{\mu} \bar{c}_{\alpha}^{a}(\tau)\right|_{\mathcal{O}\left(g^{2}\right)}+\left.c_{\alpha}^{a} \frac{d z_{\alpha}^{\mu}(\tau)}{d \tau}\right|_{\mathcal{O}\left(g^{2}\right)}\right),
\end{aligned}
$$

$\left.(1 \mathrm{~b})^{\mu a}(k) \equiv \sum_{\alpha=1}^{N} \int d \tau e^{i k \cdot\left(b_{\alpha}+v_{\alpha} \tau\right)} i k \cdot z_{\alpha}(\tau)\right|_{\mathcal{O}\left(g^{2}\right)} v_{\alpha}^{\mu} c_{\alpha}^{a}$,

$\left.(1 \mathrm{c})^{\mu a}(k) \equiv j^{\mu a}(k)\right|_{\mathcal{O}\left(g^{2}\right)}$,

$(1 \mathrm{~d})^{\mu a}(k) \equiv \sum_{\alpha=1}^{N} \int d \tau e^{i k \cdot\left(b_{\alpha}+v_{\alpha} \tau\right)}$

$\times\left(\left.v_{\alpha}^{\mu} \bar{c}_{\alpha}^{a}(\tau)\right|_{\mathcal{O}\left(y^{2}\right)}+\left.c_{\alpha}^{a} \frac{d z_{\alpha}^{\mu}(\tau)}{d \tau}\right|_{\mathcal{O}\left(y^{2}\right)}\right)$,

$\left.(1 \mathrm{e})^{\mu a}(k) \equiv \sum_{\alpha=1}^{N} \int d \tau e^{i k \cdot\left(b_{\alpha}+v_{\alpha} \tau\right)} i k \cdot z_{\alpha}(\tau)\right|_{\mathcal{O}\left(y^{2}\right)} v_{\alpha}^{\mu} c_{\alpha}^{a}$,

$\left.(1 \mathrm{f})^{\mu a}(k) \equiv j^{\mu a}(k)\right|_{\mathcal{O}\left(y^{2}\right)}$,

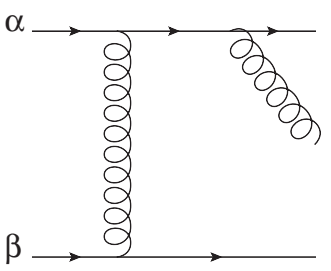

(a)

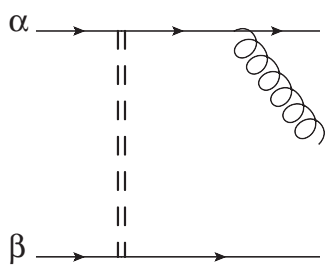

(d)

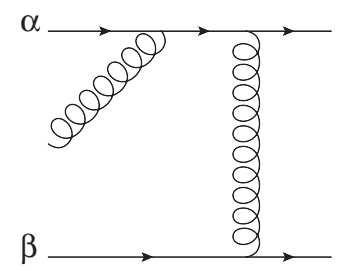

(b)

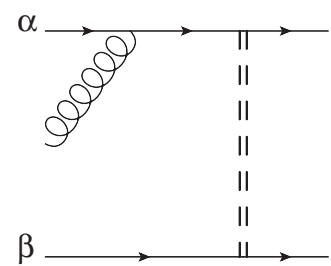

(e)

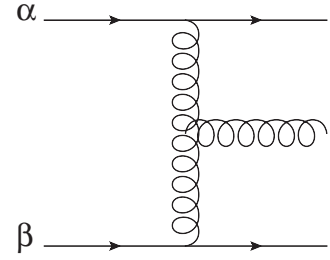

(c)

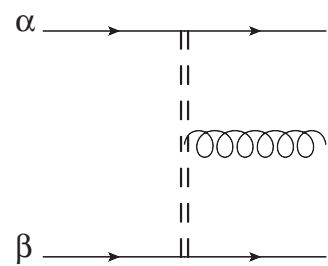

(f)

FIG. 1. These diagrams represent all of the contributions to $\hat{J}^{\mu a}$ in Yang-Mills-biadjoint-scalar theory. Straight lines represent matter fields, curly lines represent Yang-Mills fields, and double-dashed lines represent biadjoint scalar fields. Diagrams 1(a), 1(b), 1(d), and 1 (e) correspond to $J^{\mu a}(k)$, while diagrams $1(\mathrm{c})$ and 1(f) correspond to $j^{\mu a}(k)$. 
where diagrams $1(\mathrm{a}), 1(\mathrm{~b}), 1(\mathrm{~d})$, and 1(e) give $J^{\mu a}(k)$ and diagrams 1(c) and 1(f) give $j^{\mu a}(k)$, both to second order in $g$ and $y$. The source $J^{\mu a}(k)$ was split into two types of diagrams, as 1(a) represents radiation that was emitted after the particle was deflected, while 1(b) represents radiation that was emitted before the particle was deflected. As such, it is anticipated that $1(\mathrm{~b})$ and $1(\mathrm{e})$ should be proportional to the undeflected quantities $v_{\alpha}^{\mu} c_{\alpha}^{a}$, while 1(a) and 1(d) are in terms of corrections such as $\frac{d z_{\alpha}^{\mu}}{d \tau} c_{\alpha}^{a}$ and $v_{\alpha}^{\mu} \bar{c}_{\alpha}^{a}$. Diagrams 1(c) and 1(f) are computed in Appendix B from the three-point vertex with three vectors and the three-point vertex with two scalars and one vector, respectively. The six diagrams sum to give $\hat{J}^{\mu a}$ and satisfy the Ward identity $k_{\mu} \hat{J}^{\mu a}(k)=0$.

Summing up the three diagrams 1 (a) -1 (c)is algebraically equivalent to $\left.\hat{J}^{\mu a}(k)\right|_{\mathcal{O}\left(g^{2}\right)}$, giving

$$
\begin{aligned}
\left.\hat{J}^{\mu a}(k)\right|_{\mathcal{O}\left(g^{2}\right)}= & g^{2} \sum_{\substack{\alpha=1 \\
\beta \neq \alpha}}^{N} \int_{l_{\alpha}, l_{\beta}} \mu_{\alpha, \beta}(k)\left[i f^{a b c} c_{\alpha}^{b} c_{\beta}^{c}\left\{2\left(k \cdot v_{\beta}\right) v_{\alpha}^{\mu}+\left(v_{\alpha} \cdot v_{\beta}\right)\left(\frac{l_{\alpha}^{2}}{k \cdot v_{\alpha}} v_{\alpha}^{\mu}-l_{\alpha}^{\mu}\right)\right\}\right. \\
& \left.+\frac{c_{\alpha}^{b} c_{\beta}^{b}}{m_{\alpha}} \frac{l_{\alpha}^{2} c_{\alpha}^{a}}{k \cdot v_{\alpha}}\left\{v_{\alpha} \cdot v_{\beta}\left(\frac{k \cdot l_{\beta}}{k \cdot v_{\alpha}} v_{\alpha}^{\mu}-l_{\beta}^{\mu}\right)+k \cdot v_{\alpha} v_{\beta}^{\mu}-k \cdot v_{\beta} v_{\alpha}^{\mu}\right\}\right],
\end{aligned}
$$

which is the pure Yang-Mills contribution found by Ref. [41]. Summing up the three diagrams 1(d)-1(f) is equivalent to $\left.\hat{J}^{\mu a}(k)\right|_{\mathcal{O}\left(y^{2}\right)}$, giving

$$
\left.\hat{J}^{\mu a}(k)\right|_{\mathcal{O}\left(y^{2}\right)}=-y^{2} \sum_{\substack{\alpha=1 \\ \beta \neq \alpha}}^{N} c_{\alpha}^{\tilde{a}} c_{\beta}^{\tilde{a}} \int_{l_{\alpha}, l_{\beta}} \mu_{\alpha, \beta}(k)\left[\frac{c_{\alpha}^{b} c_{\beta}^{b}}{m_{\alpha}} \frac{l_{\alpha}^{2} c_{\alpha}^{a}}{k \cdot v_{\alpha}}\left(\frac{k \cdot l_{\beta}}{k \cdot v_{\alpha}} v_{\alpha}^{\mu}-l_{\beta}^{\mu}\right)+i f^{a b c} c_{\alpha}^{b} c_{\beta}^{c}\left(\frac{l_{\alpha}^{2}}{k \cdot v_{\alpha}} v_{\alpha}^{\mu}-l_{\alpha}^{\mu}\right)\right] .
$$

The radiative field must be gauge invariant and the above expression satisfies the Ward identity $\left.k_{\mu} \hat{J}^{\mu a}(k)\right|_{\mathcal{O}\left(g y^{2}\right)}=0$, as the identity must be satisfied order by order. Adding the above contributions to Eq. (2.27) gives the total source, $\hat{J}^{\mu a}$. To find the radiation field $A_{\text {rad }}^{\mu a}$ from the source $\hat{J}^{\mu a}[41,56]$,

$$
A_{\mathrm{rad}}^{\mu a}(x)=\frac{g}{4 \pi r} \int \frac{d \omega}{2 \pi} e^{-i \omega t} \hat{\jmath}^{\mu a}(k),
$$

where $k^{\mu}=\omega(1, \vec{x} / r)$.

\section{GRAVITATIONAL RADIATION IN EINSTEIN-YANG-MILLS THEORY}

\section{A. Equations of motion and initial conditions}

The action for the Einstein-Yang-Mills-dilaton theory in consideration is

$$
\begin{aligned}
S= & \int d^{d} x \sqrt{-g}\left[-\frac{2}{\kappa^{2}} R-\frac{1}{4} g^{\mu \rho} g^{\nu \sigma} F_{\mu \nu}^{\tilde{a}} F_{\rho \sigma}^{\tilde{a}}\right. \\
& \left.+\frac{2}{\kappa^{2}}(d-2) g^{\mu \nu} \partial_{\mu} \phi \partial_{\nu} \phi\right]-m \int d \tau e^{\phi},
\end{aligned}
$$

where $\phi$ is the dilaton field and $d \tau=\sqrt{g_{\mu \nu} d x^{\mu} d x^{\nu}}$. By varying the action above, the energy-momentum pseudotensor contributions from the Yang-Mills field and the dilaton are given by

$$
\begin{aligned}
8 \pi G T_{\mu \nu}= & R_{\mu \nu}-\frac{1}{2} g_{\mu \nu} R \\
& -(d-2)\left(\partial_{\mu} \phi \partial_{\nu} \phi-\frac{1}{2} g_{\mu \nu} g^{\rho \sigma} \partial_{\rho} \phi \partial_{\sigma} \phi\right) \\
& +8 \pi G\left(g^{\rho \sigma} F_{\mu \rho}^{\tilde{a}} F_{\nu \sigma}^{\tilde{a}}-\frac{1}{4} g_{\mu \nu} g^{\rho \sigma} g^{\lambda \tau} F_{\rho \lambda}^{\tilde{a}} F_{\sigma \tau}^{\tilde{a}}\right) .
\end{aligned}
$$

According to Dirac, $\sqrt{|g|} T^{\mu \nu}$ is the density and flux of energy and momentum for matter [57] such that in the presence of gravity, $N$ particles contribute

$$
\sqrt{|g|} T^{\mu \nu}(x)=\sum_{\alpha=1}^{N} m_{\alpha} \int d \tau v_{\alpha}^{\mu}(\tau) v_{\alpha}^{\nu}(\tau) \delta^{d}\left(x-x_{\alpha}(\tau)\right)
$$

A weak-field approximation is taken by introducing $h^{\mu \nu}$ as

$$
\begin{aligned}
& g_{\mu \nu}=\eta_{\mu \nu}+\kappa h_{\mu \nu}, \\
& g^{\mu \nu}=\eta^{\mu \nu}-\kappa h^{\mu \nu}+\kappa^{2} h^{\mu \rho} h_{\rho}^{\nu}+\cdots, \\
& |g| \equiv-\operatorname{det}\left(g_{\mu \nu}\right)=1+\kappa h-\frac{\kappa^{2}}{2}\left(h^{\mu \nu} h_{\mu \nu}-h^{2}\right)+\cdots,
\end{aligned}
$$

where $h \equiv h_{\rho}^{\rho}$ and the radiation can be calculated perturbatively in powers of $\kappa$. Textbook presentations of gravitational waves often focus on linearized gravity [43], which introduces the trace-reversed metric, 


$$
\bar{h}_{\mu \nu} \equiv h_{\mu \nu}-\frac{1}{2} \eta_{\mu \nu} h
$$

and find that $\square \bar{h}^{\mu \nu}=-\frac{\kappa}{2} T^{\mu \nu}$. If an effective energymomentum pseudotensor $\hat{T}^{\mu \nu}$ was found to contain contributions from matter, nonlinear gravitational field contributions, and the other fields, then the following equation of motion can be solved iteratively within the context of the weak-field approximation

$$
\square \bar{h}^{\mu \nu}=-\frac{\kappa}{2} \hat{T}^{\mu \nu} .
$$

Because of the harmonic gauge condition, the pseudotensor satisfies $\partial_{\mu} \hat{T}^{\mu \nu}=0$. The field contributions to the pseudotensor $t^{\mu \nu} \equiv \hat{T}^{\mu \nu}-T^{\mu \nu}$ will be found by expanding Eq. (3.2). The pseudotensor slightly differs from the common pseudotensor used by Landau and Lifshitz [43,47,58] and is closer to ones used previously by Einstein and Dirac, giving

$$
\hat{T}^{\mu \nu}=T^{\mu \nu}+t^{\mu \nu} \equiv \sqrt{|g|} T^{\mu \nu}+\hat{t}^{\mu \nu},
$$

where $\hat{t}^{\mu \nu}$ is conveniently defined to absorb $(1-\sqrt{|g|}) T^{\mu \nu}$. In this section, the algebraic method of perturbing Einstein's field equations and iteratively solving for the radiation field is presented, leaving some technical details of the calculation of $\hat{t}^{\mu \nu}$ to Appendix A. Since the threepoint graviton vertex is derived from the Lagrangian of the full theory, diagrams can encode how to find higher order field contributions from linearized field solutions. In Appendix B, radiative Feynman rules are provided for the diagrams contributing to $t^{\mu \nu}$.

The Christoffel symbol $\Gamma_{\mu \nu}^{\rho}$ and the Ricci tensor $R_{\mu \nu}$ are given by

$$
\begin{aligned}
\Gamma_{\mu \nu}^{\rho} & =\frac{1}{2} g^{\rho \sigma}\left(g_{\sigma \nu, \mu}+g_{\sigma \mu, \nu}-g_{\mu \nu, \sigma}\right), \\
R_{\mu \nu} & =\Gamma^{\rho}{ }_{\mu \nu, \rho}-\Gamma_{\mu \rho, \nu}^{\rho}+\Gamma^{\rho}{ }_{\sigma \rho} \Gamma_{\mu \nu}^{\sigma}-\Gamma_{\sigma \nu}^{\rho} \Gamma_{\mu \rho}^{\sigma} .
\end{aligned}
$$

After expanding the metric perturbatively in $\kappa$ and applying the gauge condition $\partial^{\mu} h_{\mu \nu}=\frac{1}{2} \eta_{\mu \nu} h^{\mu}$,

$$
\begin{aligned}
\Gamma^{\rho}{ }_{\mu \nu}= & \frac{\kappa}{2}\left(h_{\nu, \mu}^{\rho}+h_{\mu, \nu}^{\rho}-h_{\mu \nu}{ }^{\rho}-\kappa h^{\rho \sigma}\left(h_{\sigma \nu, \mu}+h_{\sigma \mu, \nu}-h_{\mu \nu, \sigma}\right)\right) \\
& +\mathcal{O}\left(\kappa^{3}\right), \\
R_{\mu \nu}= & -\frac{\kappa}{2} \square h_{\mu \nu}+\frac{\kappa^{2}}{2}\left[h^{\rho \sigma}\left(h_{\mu \nu, \rho \sigma}+h_{\rho \sigma, \mu \nu}-h_{\sigma \nu, \mu \rho}-h_{\mu \rho, \sigma \nu}\right)\right. \\
& \left.+h_{\mu \rho, \sigma} h_{\nu}^{\rho, \sigma}-h_{\mu \rho, \sigma} h_{\nu}^{\sigma, \rho}+\frac{1}{2} h_{\rho \sigma, \mu} h_{\nu}^{\rho \sigma,}\right] \\
& +\mathcal{O}\left(\kappa^{3}\right) .
\end{aligned}
$$

This gives the Ricci scalar $R$,

$$
\begin{aligned}
R= & \left(\eta^{\mu \nu}-\kappa h^{\mu \nu}\right) R_{\mu \nu} \\
= & -\frac{\kappa}{2} \square h+\kappa^{2}\left(h^{\rho \sigma} \square h_{\rho \sigma}+\frac{3}{4} h^{\rho \sigma, \mu} h_{\rho \sigma, \mu}-\frac{1}{2} h^{\mu \rho, \sigma} h_{\mu \sigma, \rho}\right) \\
& +\mathcal{O}\left(\kappa^{3}\right) .
\end{aligned}
$$

To lowest order, $R^{\mu \nu}-\frac{1}{2} g^{\mu \nu} R \approx-\frac{\kappa}{2} \bar{h}^{\mu \nu}$, and all higher order terms in Eq. (3.2) are subtracted to the other side of the equation to be absorbed into the definition of $t^{\mu \nu}$. Splitting these terms among $\left.t^{\mu \nu}\right|_{\Delta h},\left.t^{\mu \nu}\right|_{\Delta \phi}$, and $\left.t^{\mu \nu}\right|_{\Delta A}$ gives

$$
\begin{aligned}
\left.t^{\mu \nu}\right|_{\Delta h}= & 2 h_{\rho \sigma}\left(h^{\mu \rho, \nu \sigma}+h^{\nu \sigma, \mu \rho}-h^{\mu \nu, \rho \sigma}-h^{\rho \sigma, \mu \nu}\right)+h^{\mu \nu} \square h \\
& -2 h^{\mu \rho} \square h_{\rho}^{\nu}-2 h^{\nu \rho} \square h_{\rho}^{\mu}-2 h^{\mu \rho, \sigma}\left(h_{\rho, \sigma}^{\nu}-h_{\sigma, \rho}^{\nu}\right) \\
& -h^{\rho \sigma, \mu} h_{\rho \sigma,}{ }^{\nu} \\
& +\eta^{\mu \nu}\left[2 h^{\rho \sigma} \square h_{\rho \sigma}+h_{\rho \sigma, \lambda}\left(\frac{3}{2} h^{\rho \sigma, \lambda}-h^{\rho \lambda, \sigma}\right)\right], \\
\left.t^{\mu \nu}\right|_{\Delta \phi}= & (d-2)\left(\frac{2}{\kappa}\right)^{2}\left(\partial^{\mu} \phi \partial^{\nu} \phi-\frac{1}{2} \eta^{\mu \nu} \partial_{\rho} \phi \partial^{\rho} \phi\right), \\
\left.t^{\mu \nu}\right|_{\Delta A}= & -F^{\mu \rho \tilde{a}} F_{\rho}^{\nu}{ }_{\rho}^{\tilde{a}}+\frac{1}{4} \eta^{\mu \nu} F^{\rho \sigma \tilde{a}} F_{\rho \sigma}^{\tilde{a}},
\end{aligned}
$$

where it is important to raise the indices on $R_{\mu \nu}-\frac{1}{2} g_{\mu \nu} R$ with $g^{\mu \nu}$ to get all of the necessary terms.

Similar to the previous section, the position of the particle is given by $x_{\alpha}^{\mu}(\tau)$, which has deflections $z_{\alpha}^{\mu}(\tau)$ which must be calculated from the field solutions. The matter is also assumed to have a color charge $c_{\alpha}^{\tilde{a}}(\tau)$, but their corrections do not source the lowest-order gravitational radiation field. The Christoffel symbol can be used to find the force on each particle, giving

$$
\left.m_{\alpha} \frac{d^{2} z_{\alpha}^{\mu}(\tau)}{d \tau^{2}}\right|_{\Delta h}=-\Gamma_{\nu \rho}^{\mu} m_{\alpha} v_{\alpha}^{\nu} v_{\alpha}^{\rho} .
$$

The equation of motion utilized for the dilaton is

$$
\left.m_{\alpha} \frac{d^{2} z_{\alpha}^{\mu}(\tau)}{d \tau^{2}}\right|_{\Delta \phi}=m_{\alpha} v_{\alpha \nu} \partial^{\mu} \phi v_{\alpha}^{\nu}
$$

While this equation differs slightly from Ref. [41], both of our total pseudotensors agree and are the physical object that satisfies the gauge-invariant Ward identity. The force due to the gauge field is

$$
\left.m_{\alpha} \frac{d^{2} z_{\alpha}^{\mu}(\tau)}{d \tau^{2}}\right|_{\Delta A}=\tilde{g} c_{\alpha}^{\tilde{a}} F^{\mu \nu \tilde{a}} v_{\alpha \nu}(\tau) .
$$

\section{B. Solutions of the radiation fields}

Figure 2 shows nine diagrams which contribute to gravitational radiation for the Einstein-Yang-Mills theory. Algebraically, the first three diagrams for the pure gravity contributions are 


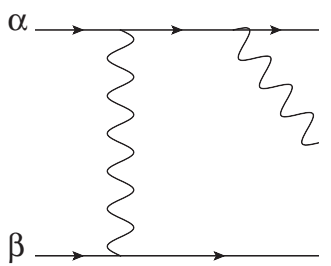

(a)

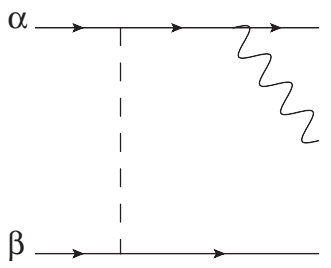

(d)

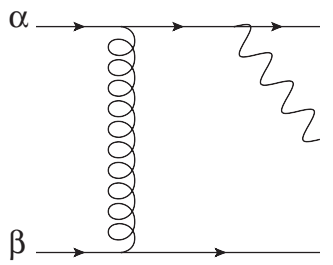

(g)

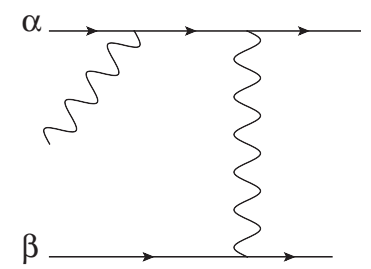

(b)

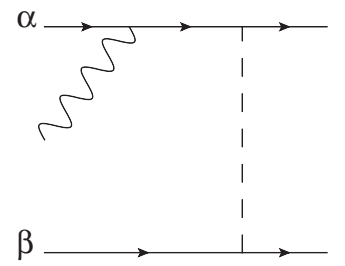

(e)

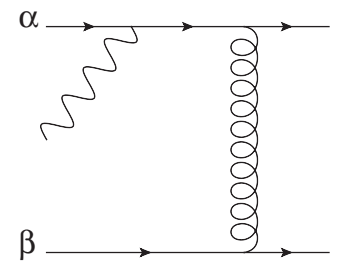

(h)

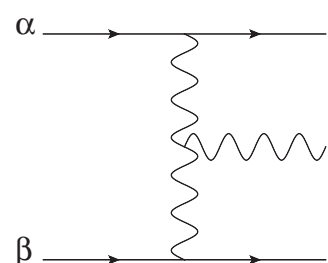

(c)

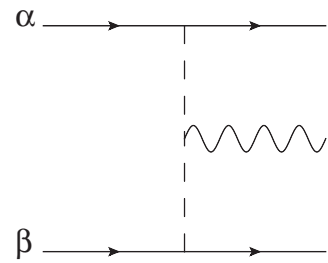

(f)

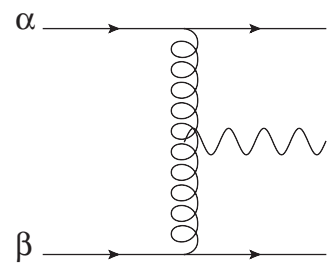

(i)

FIG. 2. These diagrams represent all of the contributions to $\hat{T}^{\mu \nu}$ in the Einstein-Yang-Mills theory. The wavy lines represent gravitational fields, the dashed lines represent dilaton fields, and the curly lines represent Yang-Mills fields. Diagrams 2(a), 2(b), 2(d), 2 (e), 2(g), and 2(h) correspond to $\sqrt{|g|} T^{\mu \nu}(k)$, while diagrams 2(c), 2(f), and 2(i) correspond to $\hat{t}^{\mu \nu}(k)$.

$$
\begin{aligned}
(2 \mathrm{a})^{\mu \nu}+(2 b)^{\mu \nu} & =\left.\sqrt{|g|} T^{\mu \nu}\right|_{\Delta h, \mathcal{O}\left(\kappa^{2}\right)} \\
(2 \mathrm{c})^{\mu \nu} & =\left.\hat{t}^{\mu \nu}\right|_{\Delta h, \mathcal{O}\left(\kappa^{2}\right)} \\
& \left.\equiv t^{\mu \nu}\right|_{\Delta h, \mathcal{O}\left(\kappa^{2}\right)}+\left.(1-\sqrt{|g|}) T^{\mu \nu}\right|_{\Delta h, \mathcal{O}\left(\kappa^{2}\right)}
\end{aligned}
$$

while the diagrams with internal dilatons algebraically represent

$$
\begin{aligned}
(2 \mathrm{~d})^{\mu \nu}+(2 \mathrm{e})^{\mu \nu} & =\left.\sqrt{|g|} T^{\mu \nu}\right|_{\Delta \phi, \mathcal{O}\left(\kappa^{2}\right)} \\
(2 \mathrm{f})^{\mu \nu} & =\left.\left.\hat{t}^{\mu \nu}\right|_{\Delta \phi, \mathcal{O}\left(\kappa^{2}\right)} \equiv t^{\mu \nu}\right|_{\Delta \phi, \mathcal{O}\left(\kappa^{2}\right)}
\end{aligned}
$$

and the diagrams with internal gauge bosons represent

$$
\begin{aligned}
(2 \mathrm{~g})^{\mu \nu}+(2 \mathrm{~h})^{\mu \nu} & =\left.\sqrt{|g|} T^{\mu \nu}\right|_{\Delta A, \mathcal{O}\left(\tilde{g}^{2}\right)} \\
(2 \mathrm{i})^{\mu \nu} & =\left.\left.\hat{t}^{\mu \nu}\right|_{\Delta A, \mathcal{O}\left(\tilde{g}^{2}\right)} \equiv t^{\mu \nu}\right|_{\Delta A, \mathcal{O}\left(\tilde{g}^{2}\right)}
\end{aligned}
$$

Since $(1-\sqrt{|g|})$ is purely gravitational, $\left.\left.\hat{t}^{\mu \nu}\right|_{\Delta \phi} \equiv t^{\mu \nu}\right|_{\Delta \phi}$ and $\left.\left.\hat{t}^{\mu \nu}\right|_{\Delta A} \equiv t^{\mu \nu}\right|_{\Delta A}$. Similar to Eq. (2.26), the diagrams 2(a), 2(b), 2(d), 2(e), 2(g), and 2(h) sum to give $\sqrt{|g|} T^{\mu \nu} ; 2(\mathrm{c})$, 2(f), and 2(i) give $\hat{t}^{\mu \nu}$; and all nine sum to give the locally conserved pseudotensor $\hat{T}^{\mu \nu}$, where all expressions are kept to second order only in $\kappa$ or $\tilde{g}$. While the diagrammatic representation may be useful for organizing higher order computations, it is simple enough to calculate $\sqrt{|g|} T^{\mu \nu}$ as a single algebraic expression for the purpose of confirming the validity of the radiative double copy to leading order.

It should be noted that an oversimplification was made, which has no physical consequences at leading order. Technically speaking, additional diagrams that couple two gravitons to an ingoing and an outgoing matter particle should be included in $t^{\mu \nu}$ for Fig. 2, as shown in Ref. [41]. However, a careful reader will note that an oversimplification was made in the computation of $T^{\mu \nu}$, since $\int d \tau$ contains an additional gravitational term due to the fact that $d \tau^{2}=g_{\mu \nu} d x^{\mu} d x^{\nu}$, which contains $\kappa$ dependence. The introduction of these higher order terms in $T^{\mu \nu}$ were found to cancel exactly with the additional diagrams needed in $t^{\mu \nu}$. The analogous diagrams with two dilaton lines coupling to matter were canceled similarly by our simplification of the dilaton force law in Eq. (3.14). These oversimplifications may not necessarily hold at higher orders.

Focusing on the energy-momentum tensor $\sqrt{|g|} T^{\mu \nu}$,

$$
\begin{aligned}
\sqrt{|g|} T^{\mu \nu}= & \sum_{\alpha=1}^{N} m_{\alpha} \int d \tau e^{i k \cdot x_{\alpha}(\tau)}\left(v_{\alpha}^{\mu}+\frac{d z_{\alpha}^{\mu}(\tau)}{d \tau}\right) \\
& \times\left(v_{\alpha}^{\nu}+\frac{d z_{\alpha}^{\nu}(\tau)}{d \tau}\right) .
\end{aligned}
$$

Solving for the appropriate field equations gives $h^{\mu \nu}, \phi$, and $A^{\mu a}$ to lowest order, 


$$
\begin{aligned}
\left.h^{\mu \nu}(x)\right|_{\mathcal{O}\left(\kappa^{1}\right)} & =\frac{\kappa}{2} \sum_{\alpha=1}^{N} m_{\alpha} \int_{l}(2 \pi) \delta\left(l \cdot v_{\alpha}\right) \frac{e^{-i l \cdot\left(x-b_{\alpha}\right)}}{l^{2}}\left(v_{\alpha}^{\mu} v_{\alpha}^{\nu}-\frac{\eta^{\mu \nu}}{d-2}\right), \\
\left.\phi(x)\right|_{\mathcal{O}\left(\kappa^{2}\right)} & =\frac{1}{(d-2)}\left(\frac{\kappa}{2}\right)^{2} \sum_{\alpha=1}^{N} m_{\alpha} \int_{l}(2 \pi) \delta\left(l \cdot v_{\alpha}\right) \frac{e^{-i l \cdot\left(x-b_{\alpha}\right)}}{l^{2}} \\
\left.A^{\mu \tilde{a}}(x)\right|_{\mathcal{O}\left(\tilde{g}^{1}\right)} & =-\tilde{g} \sum_{\alpha=1}^{N} \int_{l}(2 \pi) \delta\left(l \cdot v_{\alpha}\right) \frac{e^{-i l \cdot\left(x-b_{\alpha}\right)}}{l^{2}} c_{\alpha}^{\tilde{a}} v_{\alpha}^{\mu} .
\end{aligned}
$$

Plugging the lowest-order field solutions into Eqs. (3.13), (3.14), and (3.15) gives

$$
\begin{aligned}
\left.\frac{d^{2} z_{\alpha}^{\mu}(\tau)}{d \tau^{2}}\right|_{\Delta h} & =i\left(\frac{\kappa}{2}\right)^{2} \sum_{\beta \neq \alpha} m_{\beta} \int_{l_{\beta}}(2 \pi) \delta\left(l_{\beta} \cdot v_{\beta}\right) \frac{e^{-i l_{\beta} \cdot\left(x-b_{\beta}\right)}}{l_{\beta}^{2}}\left[2\left(v_{\alpha} \cdot v_{\beta}\right) k \cdot v_{\alpha} v_{\beta}^{\mu}-\frac{2 k \cdot v_{\alpha}}{d-2} v_{\alpha}^{\mu}-\left(\left(v_{\alpha} \cdot v_{\beta}\right)^{2}-\frac{1}{d-2}\right) l_{\beta}^{\mu}\right], \\
\left.\frac{d^{2} z_{\alpha}^{\mu}(\tau)}{d \tau^{2}}\right|_{\Delta \phi} & =\frac{-i}{d-2}\left(\frac{\kappa}{2}\right)^{2} \sum_{\beta \neq \alpha} m_{\beta} \int_{l_{\beta}}(2 \pi) \delta\left(l_{\beta} \cdot v_{\beta}\right) \frac{e^{-i l_{\beta} \cdot\left(x-b_{\beta}\right)}}{l_{\beta}^{2}} l_{\beta}^{\mu}, \\
\left.\frac{d^{2} z_{\alpha}^{\mu}}{d \tau^{2}}\right|_{\Delta A} & =i \tilde{g}^{2} \sum_{\beta \neq \alpha} \frac{c_{\alpha}^{\tilde{a}} c_{\beta}^{\tilde{a}}}{m_{\alpha}} \int_{l}(2 \pi) \delta\left(l \cdot v_{\beta}\right) \frac{e^{-i l \cdot\left(b_{\alpha \beta}+v_{\alpha} \tau\right)}}{l^{2}}\left[\left(v_{\alpha} \cdot v_{\beta}\right) l^{\mu}-\left(l \cdot v_{\alpha}\right) v_{\beta}^{\mu}\right] .
\end{aligned}
$$

The corrections to the position are useful for finding $\sqrt{|g|} T^{\mu \nu}(k)$,

$$
\begin{aligned}
\sqrt{|g|} T^{\mu \nu}(k) & =\sum_{\alpha=1}^{N} m_{\alpha} \int d \tau e^{i k \cdot\left(b_{\alpha}+v_{\alpha} \tau+z_{\alpha}(\tau)\right)}\left(v_{\alpha}^{\mu}+\frac{d z_{\alpha}^{\mu}(\tau)}{d \tau}\right)\left(v_{\alpha}^{\nu}+\frac{d z_{\alpha}^{\nu}(\tau)}{d \tau}\right), \\
\left.\sqrt{|g|} T^{\mu \nu}(k)\right|_{\mathcal{O}\left(\kappa^{2}\right)} & =\sum_{\alpha=1}^{N} m_{\alpha} \int d \tau e^{i k \cdot\left(b_{\alpha}+v_{\alpha} \tau\right)}\left[i k \cdot z_{\alpha} v_{\alpha}^{\mu} v_{\alpha}^{\nu}+\frac{d z_{\alpha}^{\mu}}{d \tau} v_{\alpha}^{\nu}+v_{\alpha}^{\mu} \frac{d z_{\alpha}^{\nu}}{d \tau}\right]
\end{aligned}
$$

The lowest order term proportional to $v_{\alpha}^{\mu} v_{\alpha}^{\nu}$ may be dropped, as it was used to find the solution to $h^{\mu \nu}$ in Eq. (3.20). Focusing on the corrections due to gravity,

$$
\begin{aligned}
\left.\sqrt{|g|} T^{\mu \nu}(k)\right|_{\Delta h}= & \left(\frac{\kappa}{2}\right)^{2} \sum_{\alpha \neq \beta} m_{\alpha} m_{\beta} \int_{l_{\alpha}, l_{\beta}} \mu_{\alpha, \beta}(k) l_{\alpha}^{2}\left[v_{\alpha}^{\mu} v_{\alpha}^{\nu}\left(2 v_{\alpha} \cdot v_{\beta} \frac{k \cdot v_{\beta}}{k \cdot v_{\alpha}}+\frac{2}{d-2}-\frac{l_{\beta} \cdot k}{\left(k \cdot v_{\alpha}\right)^{2}}\left(\left(v_{\alpha} \cdot v_{\beta}\right)^{2}-\frac{1}{d-2}\right)\right)\right. \\
& \left.-2 v_{\alpha} \cdot v_{\beta}\left(v_{\alpha}^{\mu} v_{\beta}^{\nu}+v_{\alpha}^{\nu} v_{\beta}^{\mu}\right)+\frac{1}{k \cdot v_{\alpha}}\left(\left(v_{\alpha} \cdot v_{\beta}\right)^{2}-\frac{1}{d-2}\right)\left(v_{\alpha}^{\mu} l_{\beta}^{\nu}+v_{\alpha}^{\nu} l_{\beta}^{\mu}\right)\right] .
\end{aligned}
$$

Additionally, the dilaton contributes

$$
\left.\sqrt{|g|} T^{\mu \nu}(k)\right|_{\Delta \phi}=\frac{1}{d-2}\left(\frac{\kappa}{2}\right)^{2} \sum_{\alpha \neq \beta} m_{\alpha} m_{\beta} \int_{l_{\alpha}, l_{\beta}} \mu_{\alpha, \beta}(k) l_{\alpha}^{2}\left[-v_{\alpha}^{\mu} v_{\alpha}^{\nu}\left(\frac{l_{\beta} \cdot k}{\left(k \cdot v_{\alpha}\right)^{2}}\right)+\frac{1}{k \cdot v_{\alpha}}\left(v_{\alpha}^{\mu} l_{\beta}^{\nu}+v_{\alpha}^{\nu} l_{\beta}^{\mu}\right)\right] .
$$

Finally, the gauge boson contributes

$$
\begin{aligned}
\left.\sqrt{|g|} T^{\mu \nu}(k)\right|_{\Delta A}= & \tilde{g}^{2} \sum_{\substack{\alpha=1 \\
\beta \neq \alpha}}^{N} c_{\alpha}^{\tilde{a}} c_{\beta}^{\tilde{a}} \int_{l_{\alpha}, l_{\beta}} \mu_{\alpha, \beta}(k) \frac{l_{\alpha}^{2}}{k \cdot v_{\alpha}} \\
& \times\left[v_{\alpha}^{\mu} v_{\alpha}^{\nu}\left(v_{\alpha} \cdot v_{\beta} \frac{k \cdot l_{\beta}}{k \cdot v_{\alpha}}-k \cdot v_{\beta}\right)+\left(v_{\alpha}^{\mu} v_{\beta}^{\nu}+v_{\alpha}^{\nu} v_{\beta}^{\mu}\right)\left(k \cdot v_{\alpha}\right)-\left(v_{\alpha}^{\mu} l_{\beta}^{\nu}+v_{\alpha}^{\nu} l_{\beta}^{\mu}\right)\left(v_{\alpha} \cdot v_{\beta}\right)\right] .
\end{aligned}
$$


Appendix A works out the algebraic details of computing $\left.t^{\mu \nu}\right|_{\Delta h}$ and $\left.\hat{t}^{\mu \nu}\right|_{\Delta h}$. In summary, gravity contributes

$$
\begin{aligned}
\left.\hat{t}^{\mu \nu}(k)\right|_{\Delta h}= & \left(\frac{\kappa}{2}\right)^{2} \sum_{\substack{\alpha=1 \\
\beta \neq \alpha}}^{N} m_{\alpha} m_{\beta} \int_{l_{\alpha}, l_{\beta}} \mu_{\alpha, \beta}(k)\left[2 v_{\alpha}^{\mu} v_{\alpha}^{\nu}\left(\left(k \cdot v_{\beta}\right)^{2}-\frac{l_{\alpha}^{2}}{d-2}\right)\right. \\
& +\left(v_{\alpha}^{\mu} v_{\beta}^{\nu}+v_{\alpha}^{\nu} v_{\beta}^{\mu}\right)\left(l_{\alpha}^{2} v_{\alpha} \cdot v_{\beta}-k \cdot v_{\alpha} k \cdot v_{\beta}\right)-2\left(v_{\alpha}^{\mu} l_{\alpha}^{\nu}+v_{\alpha}^{\nu} l_{\alpha}^{\mu}\right)\left(v_{\alpha} \cdot v_{\beta} k \cdot v_{\beta}\right) \\
& \left.+l_{\alpha}^{\mu} l_{\alpha}^{\mu}\left(\left(v_{\alpha} \cdot v_{\beta}\right)^{2}-\frac{1}{d-2}\right)+\eta^{\mu \nu}\left(k \cdot v_{\alpha} k \cdot v_{\beta} v_{\alpha} \cdot v_{\beta}-\frac{l_{\alpha}^{2}}{2}\left(\left(v_{\alpha} \cdot v_{\beta}\right)^{2}-\frac{1}{d-2}\right)\right)\right] .
\end{aligned}
$$

Similarly, Eq. (3.12) the dilaton contributes

$$
\left.\hat{t}^{\mu \nu}(k)\right|_{\Delta \phi}=\frac{1}{(d-2)}\left(\frac{\kappa}{2}\right)^{2} \sum_{\substack{\alpha=1 \\ \beta \neq \alpha}}^{N} m_{\alpha} m_{\beta} \int_{l_{\alpha}, l_{\beta}} \mu_{\alpha, \beta}(k)\left[-l_{\alpha}^{\mu} l_{\beta}^{\nu}+\eta^{\mu \nu} \frac{l_{\alpha} \cdot l_{\beta}}{2}\right] .
$$

When calculated algebraically from Eq. (3.12), the gauge boson contributes

$$
\begin{aligned}
\left.\hat{t}^{\mu \nu}(k)\right|_{\Delta A}= & \tilde{g}^{2} \sum_{\substack{\alpha=1 \\
\beta \neq \alpha}}^{N} c_{\alpha}^{\tilde{a}} c_{\beta}^{\tilde{a}} \int_{l_{\alpha}, l_{\beta}} \mu_{\alpha, \beta}(k)\left[\frac{1}{2}\left(v_{\alpha}^{\mu} v_{\beta}^{\nu}+v_{\alpha}^{\nu} v_{\alpha}^{\mu}\right) l_{\alpha} \cdot l_{\beta}+\left(v_{\alpha}^{\mu} l_{\alpha}^{\nu}+v_{\alpha}^{\nu} l_{\alpha}^{\mu}\right) k \cdot v_{\beta}-l_{\alpha}^{\mu} l_{\alpha}^{\nu} v_{\alpha} \cdot v_{\beta}\right. \\
& \left.-\frac{1}{2} \eta^{\mu \nu}\left(k \cdot v_{\alpha} k \cdot v_{\beta}+v_{\alpha} \cdot v_{\beta} l_{\alpha} \cdot l_{\beta}\right)\right] .
\end{aligned}
$$

In Appendix B, the three-point boson vertices of the Einstein-Yang-Mills theory are used to find the same results for $t^{\mu \nu}$ via radiative Feynman rules.

Summing all contributions to order $\kappa^{2}$ gives the contributions from pure dilaton gravity,

$$
\begin{aligned}
\left.\hat{T}^{\mu \nu}(k)\right|_{\mathcal{O}\left(\kappa^{2}\right)}= & \left(\frac{\kappa}{2}\right)^{2} \sum_{\substack{\alpha=1 \\
\beta \neq \alpha}}^{N} m_{\alpha} m_{\beta} \int_{l_{\alpha}, l_{\beta}} \mu_{\alpha, \beta}(k)\left[v_{\alpha}^{\mu} v_{\alpha}^{\nu}\left(2\left(k \cdot v_{\beta}\right)^{2}+2 k \cdot v_{\beta} \frac{l_{\alpha}^{2} v_{\alpha} \cdot v_{\beta}}{k \cdot v_{\alpha}}-\frac{l_{\alpha}^{2}\left(v_{\alpha} \cdot v_{\beta}\right)^{2} k \cdot l_{\beta}}{\left(k \cdot v_{\alpha}\right)^{2}}\right)\right. \\
& -\left(v_{\alpha}^{\mu} v_{\beta}^{\nu}+v_{\alpha}^{\nu} v_{\beta}^{\mu}\right)\left(l_{\alpha}^{2} v_{\alpha} \cdot v_{\beta}+k \cdot v_{\alpha} k \cdot v_{\beta}\right)-\left(v_{\alpha}^{\mu} l_{\alpha}^{\nu}+v_{\alpha}^{\nu} l_{\alpha}^{\mu}\right)\left(v_{\alpha} \cdot v_{\beta}\right)\left(\frac{l_{\alpha}^{2} v_{\alpha} \cdot v_{\beta}}{k \cdot v_{\alpha}}+2 k \cdot v_{\beta}\right) \\
& \left.+l_{\alpha}^{\mu} l_{\alpha}^{\nu}\left(v_{\alpha} \cdot v_{\beta}\right)^{2}+\eta^{\mu \nu}\left(v_{\alpha} \cdot v_{\beta}\right)\left(\frac{l_{\alpha}^{2} v_{\alpha} \cdot v_{\beta}}{2}+k \cdot v_{\alpha} k \cdot v_{\beta}\right)\right],
\end{aligned}
$$

which agree with the results found in Ref. [41]. $\left.\sqrt{|g|} T^{\mu \nu}\right|_{\Delta A}$ and $\left.\hat{t}^{\mu \nu}\right|_{\Delta A}$ give the additional contributions in the EinsteinYang-Mills theory,

$$
\begin{aligned}
\left.\hat{T}^{\mu \nu}(k)\right|_{\mathcal{O}\left(\tilde{g}^{2}\right)}= & \tilde{g}^{2} \sum_{\substack{\alpha=1 \\
\beta \neq \alpha}}^{N} \int_{l_{\alpha}, l_{\beta}} \mu_{\alpha, \beta}(k)\left[v_{\alpha}^{\mu} v_{\alpha}^{\nu}\left(v_{\alpha} \cdot v_{\beta} \frac{k \cdot l_{\beta}}{\left(k \cdot v_{\alpha}\right)^{2}}-\frac{k \cdot v_{\beta}}{k \cdot v_{\alpha}}\right) l_{\alpha}^{2}+\frac{1}{2}\left(v_{\alpha}^{\mu} v_{\beta}^{\nu}+v_{\alpha}^{\nu} v_{\beta}^{\mu}\right) l_{\alpha}^{2}\right. \\
& \left.+\left(v_{\alpha}^{\mu} l_{\alpha}^{\nu}+v_{\alpha}^{\nu} l_{\alpha}^{\mu}\right)\left(\frac{l_{\alpha}^{2} v_{\alpha} \cdot v_{\beta}}{k \cdot v_{\alpha}}+k \cdot v_{\beta}\right)-l_{\alpha}^{\mu} l_{\alpha}^{\nu} v_{\alpha} \cdot v_{\beta}-\frac{1}{2} \eta^{\mu \nu}\left(k \cdot v_{\alpha} k \cdot v_{\beta}+l_{\alpha}^{2} v_{\alpha} \cdot v_{\beta}\right)\right] .
\end{aligned}
$$

Adding this result to Eq. (3.29) gives the total source for gravitational radiation for the Einstein-Yang-Mills theory. Next, we show that this result agrees precisely with what is found with the radiative double-copy method.

\section{The radiative double copy}

In order to use the double copy to find gravitational radiation in the Einstein-Yang-Mills theory, the same replacement rules used for general relativity [41] may be used with the radiation found in the Yang-Mills-biadjoint-scalar theory. The replacement rules are 


$$
\begin{aligned}
g \rightarrow & \frac{\kappa}{2}, \quad y \rightarrow \tilde{g}, \quad c_{\alpha}^{a} \rightarrow p_{\alpha}^{\nu}, \\
i f^{a_{1} a_{2} a_{3}} \rightarrow & -\frac{1}{2}\left(\eta^{\nu_{1} \nu_{3}}\left(q_{1}-q_{3}\right)^{\nu_{2}}+\eta^{\nu_{1} \nu_{2}}\left(q_{2}-q_{1}\right)^{\nu_{3}}\right. \\
& \left.+\eta^{\nu_{2} \nu_{3}}\left(q_{3}-q_{2}\right)^{\nu_{1}}\right), \\
\hat{J}^{\mu a}(k) \rightarrow & \hat{T}^{\mu \nu}(k),
\end{aligned}
$$

where the momenta $q_{1}+q_{2}+q_{3}=0$. Similar to the Ward identity $k_{\mu} \hat{\jmath}^{\mu a}=0$, we can shift $\hat{T}^{\mu \nu}$ by terms proportional to either $k^{\mu}$ or $k^{\nu}$, such that $k_{\mu} \hat{T}^{\mu \nu}=$ $k_{\nu} \hat{T}^{\mu \nu}=0$, which shifts the gauge-dependent pseudotensor into the harmonic gauge. Since Ref. [41] showed that the radiative double copy could recover $\left.\hat{T}^{\mu \nu}\right|_{\mathcal{O}\left(\kappa^{2}\right)}$ and Ref. [40] showed how to use Yang-Mills ghosts to remove the dilaton, we focus on the additional terms introduced in the Einstein-Yang-Mills theory. Applying the double copy replacement rules in Eq. (3.31) to Eq. (2.29) gives

$$
\begin{aligned}
\left.\hat{T}^{\mu \nu}(k)\right|_{\tilde{g}^{2}}= & \tilde{g}^{2} \sum_{\substack{\alpha=1 \\
\beta \neq \alpha}}^{N} m_{\alpha} m_{\beta} c_{\alpha}^{\tilde{a}} c_{\beta}^{\tilde{a}} \int_{l_{\alpha}, l_{\beta}} \mu_{\alpha, \beta}(k)\left[v_{\alpha} \cdot v_{\beta} \frac{l_{\alpha}^{2} v_{\alpha}^{\nu}}{k \cdot v_{\alpha}}\left(\frac{k \cdot l_{\beta}}{k \cdot v_{\alpha}} v_{\alpha}^{\mu}-l_{\beta}^{\mu}\right)\right. \\
& \left.-\frac{1}{2}\left(2 k \cdot v_{\beta} v_{\alpha}^{\nu}-2 k \cdot v_{\alpha} v_{\beta}^{\nu}+v_{\alpha} \cdot v_{\beta}\left(l_{\beta}-l_{\alpha}\right)^{\nu}\right)\left(\frac{l_{\alpha}^{2}}{k \cdot v_{\alpha}} v_{\alpha}^{\mu}-l_{\alpha}^{\mu}\right)\right] .
\end{aligned}
$$

Shifting $l_{\beta}^{\mu} \rightarrow\left(l_{\beta}-l_{\alpha}\right)^{\mu} / 2$ gives the gauge invariant $\hat{T}^{\mu \nu}$,

$$
\begin{aligned}
\left.\hat{T}^{\mu \nu}(k)\right|_{\mathcal{O}\left(\tilde{g}^{2}\right)=} & \tilde{g}^{2} \sum_{\substack{\alpha=1 \\
\beta \neq \alpha}}^{N} m_{\alpha} m_{\beta} c_{\alpha}^{\tilde{a}} c_{\beta}^{\tilde{a}} \int_{l_{\alpha}, l_{\beta}} \mu_{\alpha, \beta}(k)\left[v_{\alpha} \cdot v_{\beta} \frac{l_{\alpha}^{2} v_{\alpha}^{\nu}}{k \cdot v_{\alpha}}\left(\frac{k \cdot l_{\beta}}{k \cdot v_{\alpha}} v_{\alpha}^{\mu}-\frac{1}{2}\left(l_{\beta}-l_{\alpha}\right)^{\mu}\right)\right. \\
& \left.-\frac{1}{2}\left(2 k \cdot v_{\beta} v_{\alpha}^{\nu}-2 k \cdot v_{\alpha} v_{\beta}^{\nu}+v_{\alpha} \cdot v_{\beta}\left(l_{\beta}-l_{\alpha}\right)^{\nu}\right)\left(\frac{l_{\alpha}^{2}}{k \cdot v_{\alpha}} v_{\alpha}^{\mu}-l_{\alpha}^{\mu}\right)\right] .
\end{aligned}
$$

Symmetrizing this result gives the appropriate final expression for $\hat{T}^{\mu \nu}$,

$$
\begin{aligned}
\left.\hat{T}^{\mu \nu}\right|_{\mathcal{O}\left(\tilde{g}^{2}\right)}= & -\tilde{g}^{2}\left(\frac{\kappa}{2}\right)^{2} \sum_{\substack{\alpha=1 \\
\beta \neq \alpha}}^{N} m_{\alpha} m_{\beta} c_{\alpha}^{\tilde{a}} c_{\beta}^{\tilde{a}} \int_{l_{\alpha}, l_{\beta}} \mu_{\alpha, \beta}(k)\left[v_{\alpha}^{\mu} v_{\alpha}^{\nu}\left(\frac{k \cdot v_{\beta}}{k \cdot v_{\alpha}}-\frac{v_{\alpha} \cdot v_{\beta}}{\left(k \cdot v_{\alpha}\right)^{2}} k \cdot l_{\beta}\right) l_{\alpha}^{2}-\frac{1}{2}\left(v_{\alpha}^{\mu} v_{\beta}^{\nu}+v_{\alpha}^{\nu} v_{\beta}^{\mu}\right) l_{\alpha}^{2}\right. \\
& \left.-\left(v_{\alpha}^{\mu} l_{\alpha}^{\nu}+v_{\alpha}^{\nu} l_{\alpha}^{\mu}\right)\left(\frac{v_{\alpha} \cdot v_{\beta}}{k \cdot v_{\alpha}} l_{\alpha}^{2}+k \cdot v_{\beta}\right)+l_{\alpha}^{\mu} l_{\alpha}^{\nu}\left(v_{\alpha} \cdot v_{\beta}\right)+\frac{1}{2} \eta^{\mu \nu} l_{\alpha}^{2}\left(v_{\alpha} \cdot v_{\beta}\right)\right]
\end{aligned}
$$

where the gauge condition allows for $v_{\alpha}^{\mu} k^{\nu}=\frac{1}{2} \eta^{\mu \nu} k \cdot v_{\alpha}$. This result agrees precisely with what was found in Eq. (3.31), demonstrating that the radiative double copy holds for the Einstein-Yang-Mills theory to leading order.

\section{Einstein-Maxwell theory}

Since it is more physically relevant to scatter massive point particles with electric charge rather than particles with weak-isospin or color, an Abelian $U(1)$ gauge symmetry is also worth studying. The action for fields in the EinsteinMaxwell theory is

$$
S=\int d^{d} x \sqrt{|g|}\left(-\frac{2}{\kappa^{2}} R-\frac{1}{4} g^{\mu \rho} g^{\nu \sigma} F_{\mu \nu} F_{\rho \sigma}\right)
$$

When comparing with the Einstein-Yang-Mills theory, the Maxwell field $A^{\mu}$ can be recovered from a single component of the Yang-Mills field $A^{\mu \tilde{a}}$. In order to find results in the Einstein-Maxwell theory from the EinsteinYang-Mills theory, care must be taken with the coupling constants. For example, the Maxwell current density for point particles is given by

$$
J^{\mu}(x)=e \sum_{\alpha=1}^{N} q_{\alpha} \int d \tau v_{\alpha}^{\mu}(\tau) \delta^{d}\left(x-x_{\alpha}(\tau)\right)
$$

where $q_{\alpha}=-1$ for electrons, such that $e q_{\alpha}$ represents the electric charge of particle $\alpha$. In order to recover the Einstein-Maxwell theory from Einstein-Yang-Mills, one must substitute $\tilde{g} \rightarrow e$ and $c_{\alpha}^{\tilde{a}} \rightarrow q_{\alpha}$, given our conventions for $\tilde{g}$ and the normalization of the Lagrangian given in Eq. (3.1). Applying these substitutions to Eq. (3.31) would give gravitational radiation in Einstein-Maxwell theory. At higher orders, $f^{\tilde{a} \tilde{b} \tilde{c}}$ would be sent to zero as well. 
In terms of the radiative double copy, an adjoint scalar field $\Phi^{a}$ could also be seen as a single component of the biadjoint scalar field $\Phi^{\tilde{a} a}$. Results for the Yang-Millsadjoint-scalar theory can easily be found from Eq. (2.29) by properly sending $c_{\alpha}^{\tilde{a}} \rightarrow q_{\alpha}$ and reinterpreting $y$ as the coupling constant of the adjoint scalar theory. It is straightforward to see that the double copy of YangMills-adjoint-scalar theory gives solutions in EinsteinMaxwell theory with the replacement rules shown in Eq. (3.31) and $y \rightarrow e$.

\section{CONCLUSIONS}

In previous work, the double copy has been applied to gravitational radiation in general relativity with a dilaton, which suggested that schematic radiative diagrams may be useful for depicting sources of radiation [41]. Similarly, it was shown that the same replacement rules can be used to find Yang-Mills radiation from biadjoint-scalar radiation [42] and that ghosts can be used to remove the dilaton [40].

In this work, the gravitational radiation produced by colliding color charges was found within the context of the Einstein-Yang-Mills theory. Our primary result demonstrates that the double copy can be used to find radiation in the Einstein-Yang-Mills theory from YangMills-biadjoint-scalar theory. These calculations provided insight on how a radiative diagrammatic scheme closer to Feynman diagrams used for scattering amplitudes may be possible. Furthermore, radiation in Einstein-Maxwell theory can be found via similar methods. This work suggests that it may be possible to develop systematic rules for constructing radiative diagrams that can be used to calculate radiation to higher orders, at least for initial conditions associated with $N$ particle scattering. It appears that rules for worldline propagators would be needed, in addition to the typical rules used for scattering amplitudes.

In future work, it would be interesting to investigate if the radiative double copy holds for higher orders, as the precise replacement rules are not yet known. Additional efforts to perform the integrals are also needed. The gravitational interactions between the quantized spin of Dirac particles would be an interesting theoretical challenge, while considering the scattering of macroscopic mass distributions with classical angular momentum would be more applicable for experiments such as LIGO. Studying the formation of bound states due to higher order effects would also be important.

\section{ACKNOWLEDGMENTS}

The author would like to thank Zvi Bern for constant guidance, as well as Walter Goldberger, Donal O'Connell, Alexander Ridgway, Jedidiah Thompson, and Julio ParraMartinez for various discussions.

\section{APPENDIX A: DERIVATION OF GRAVITATIONAL RADIATION FROM PSEUDOTENSOR}

In this section, the steps for deriving the gravitational radiation coming from nonlinear gravitational interactions are provided. In Sec. III, Einstein's field equations to first order for weak gravitational fields was found to be

$$
\square \bar{h}^{\mu \nu}=-\frac{\kappa}{2} \hat{T}^{\mu \nu},
$$

where the energy-momentum pseudotensor $\hat{T}^{\mu \nu}=$ $T^{\mu \nu}+t^{\mu \nu}=\sqrt{|g|} T^{\mu \nu}+\hat{t}^{\mu \nu}$ contains the nonlinear corrections to the linearized field equations, such that the purely gravitational component of the pseudotensor $t^{\mu \nu}$ is given by Eq. (3.12)

$$
\begin{aligned}
t^{\mu \nu}= & 2 h_{\rho \sigma}\left(h^{\mu \rho, \nu \sigma}+h^{\nu \sigma, \mu \rho}-h^{\mu \nu, \rho \sigma}-h^{\rho \sigma, \mu \nu}\right)+h^{\mu \nu} \square h \\
& -2 h^{\mu \rho} \square h_{\rho}^{\nu}-2 h^{\nu \rho} \square h_{\rho}^{\mu}-2 h^{\mu \rho, \sigma}\left(h_{\rho, \sigma}^{\nu}-h_{\sigma, \rho}^{\nu}\right) \\
& -h^{\rho \sigma, \mu} h_{\rho \sigma}^{\nu}+\eta^{\mu \nu}\left[2 h^{\rho \sigma} \square h_{\rho \sigma}+h_{\rho \sigma, \lambda}\left(\frac{3}{2} h^{\rho \sigma, \lambda}-h^{\rho \lambda, \sigma}\right)\right] .
\end{aligned}
$$

In order to solve for this, the lowest-order solution of the gravitational field is used,

$$
\begin{aligned}
h^{\mu \nu}(x)= & \frac{\kappa}{2} \sum_{\alpha=1}^{N} m_{\alpha} \int_{l_{\alpha}}(2 \pi) \delta\left(l_{\alpha} \cdot v_{\alpha}\right) \frac{e^{-i l_{\alpha} \cdot\left(x-b_{\alpha}\right)}}{l_{\alpha}^{2}} \\
& \times\left(v_{\alpha}^{\mu} v_{\alpha}^{\nu}-\frac{\eta^{\mu \nu}}{d-2}\right),
\end{aligned}
$$

which gives rise to a source for the nonlinear gravitational interaction via $t^{\mu \nu}$. Each term in $t^{\mu \nu}$ is second order in $h^{\mu \nu}$, so one is related to particle $\alpha$ and another to particle $\beta$, giving a double sum. The summation and integrals on all terms will have the following form:

$$
t^{\mu \nu}=\left(\frac{\kappa}{2}\right)^{2} \sum_{\substack{\alpha=1 \\ \beta \neq \alpha}}^{N} m_{\alpha} m_{\beta} \int_{l_{\alpha}, l_{\beta}} \mu_{\alpha, \beta}(k) I^{\mu \nu},
$$

where $I^{\mu \nu}$ is the integrand containing many terms. For the integrand, focusing on the $\left(v_{\alpha}^{\mu} v_{\alpha}^{\nu}-\eta^{\mu \nu} /(d-2)\right)$ portion of the solution to $h^{\mu \nu}$ and manually plugging these pieces into Eq. (A2) gives 


$$
\begin{aligned}
I^{\mu \nu}= & 2\left(v_{\beta \rho} v_{\beta \sigma}-\frac{\eta_{\rho \sigma}}{d-2}\right)\left[-l_{\alpha}^{\nu} l_{\alpha}^{\sigma}\left(v_{\alpha}^{\mu} v_{\alpha}^{\rho}-\frac{\eta^{\mu \rho}}{d-2}\right)-l_{\alpha}^{\mu} l_{\alpha}^{\rho}\left(v_{\alpha}^{\nu} v_{\alpha}^{\sigma}-\frac{\eta^{\nu \sigma}}{d-2}\right)+l_{\alpha}^{\rho} l_{\alpha}^{\sigma}\left(v_{\alpha}^{\mu} v_{\alpha}^{\nu}-\frac{\eta^{\mu \nu}}{d-2}\right)+l_{\alpha}^{\mu} l_{\alpha}^{\nu}\left(v_{\alpha}^{\rho} v_{\alpha}^{\sigma}-\frac{\eta^{\rho \sigma}}{d-2}\right)\right] \\
& -l_{\beta}^{2}\left(v_{\alpha}^{\mu} v_{\alpha}^{\nu}-\frac{\eta^{\mu \nu}}{d-2}\right)\left(\frac{-2}{d-2}\right)+2 l_{\alpha}^{2}\left(v_{\beta}^{\mu} v_{\beta}^{\rho}-\frac{\eta^{\mu \rho}}{d-2}\right)\left(v_{\alpha \rho} v_{\alpha}^{\nu}-\frac{\eta_{\rho}^{\nu}}{d-2}\right)+2 l_{\alpha}^{2}\left(v_{\beta}^{\nu} v_{\beta}^{\rho}-\frac{\eta^{\nu \rho}}{d-2}\right)\left(v_{\alpha \rho} v_{\alpha}^{\mu}-\frac{\eta_{\rho}^{\mu}}{d-2}\right) \\
& -2 i l_{\alpha}^{\sigma}\left(v_{\alpha}^{\mu} v_{\alpha}^{\rho}-\frac{\eta^{\mu \rho}}{d-2}\right)\left[i l_{\beta \sigma}\left(v_{\beta}^{\nu} v_{\beta \rho}-\frac{\eta_{\rho}^{\nu}}{d-2}\right)-i l_{\beta \rho}\left(v_{\beta}^{\nu} v_{\beta \sigma}-\frac{\eta_{\sigma}^{\nu}}{d-2}\right)\right]+l_{\alpha}^{\mu} l_{\beta}^{\nu}\left(v_{\alpha}^{\rho} v_{\alpha}^{\sigma}-\frac{\eta^{\rho \sigma}}{d-2}\right)\left(v_{\beta \rho} v_{\beta \sigma}-\frac{\eta_{\rho \sigma}}{d-2}\right) \\
& +\eta^{\mu \nu}\left\{-2 l_{\alpha}^{2}\left(v_{\alpha}^{\rho} v_{\alpha}^{\sigma}-\frac{\eta^{\rho \sigma}}{d-2}\right)\left(v_{\beta \rho} v_{\beta \sigma}-\frac{\eta_{\rho \sigma}}{d-2}\right)+i l_{\alpha \lambda}\left(v_{\alpha \rho} v_{\alpha \sigma}-\frac{\eta_{\rho \sigma}}{d-2}\right)\left[\frac{3}{2} i l_{\beta}^{\lambda}\left(v_{\beta}^{\rho} v_{\beta}^{\sigma}-\frac{\eta^{\rho \sigma}}{d-2}\right)-i l_{\beta}^{\sigma}\left(v_{\beta}^{\rho} v_{\beta}^{\lambda}-\frac{\eta^{\rho \lambda}}{d-2}\right)\right]\right\} .
\end{aligned}
$$

Distributing these factors and reorganizing all of the terms with the same tensor index structure gives

$$
\begin{aligned}
I^{\mu \nu}= & v_{\alpha}^{\mu} v_{\alpha}^{\nu}\left(2\left(k \cdot v_{\beta}\right)^{2}-\frac{2 l_{\alpha}^{2}}{d-2}+\frac{2 l_{\beta}^{2}}{d-2}-\frac{4 l_{\alpha}^{2}+4 l_{\beta}^{2}}{d-2}-\frac{4 l_{\alpha} \cdot l_{\beta}}{d-2}\right)+\left(v_{\alpha}^{\mu} v_{\beta}^{\nu}+v_{\alpha}^{\nu} v_{\beta}^{\mu}\right)\left(2 l_{\alpha}^{2}\left(v_{\alpha} \cdot v_{\beta}\right)+l_{\alpha} \cdot l_{\beta}\left(v_{\alpha} \cdot v_{\beta}\right)\right. \\
& \left.-k \cdot v_{\alpha} k \cdot v_{\beta}\right)+\left(v_{\alpha}^{\mu} l_{\alpha}^{\nu}+v_{\alpha}^{\nu} l_{\alpha}^{\mu}\right)\left(-2\left(v_{\alpha} \cdot v_{\beta}\right) k \cdot v_{\beta}+\frac{2 k \cdot v_{\alpha}}{d-2}\right)+\left(v_{\alpha}^{\mu} l_{\beta}^{\nu}+v_{\alpha}^{\nu} l_{\beta}^{\mu}\right)\left(\frac{2 k \cdot v_{\alpha}}{d-2}\right) \\
& +l_{\alpha}^{\mu} l_{\alpha}^{\nu}\left(-\frac{4}{(d-2)^{2}}+2\left(v_{\alpha} \cdot v_{\beta}\right)^{2}-\frac{4}{d-2}+\frac{2 d}{(d-2)^{2}}\right)+\left(l_{\alpha}^{\mu} l_{\beta}^{\nu}+l_{\alpha}^{\nu} l_{\beta}^{\mu}\right)\left(-\frac{1}{(d-2)^{2}}+\frac{1}{2}\left(v_{\alpha} \cdot v_{\beta}\right)^{2}-\frac{1}{d-2}+\frac{d}{2(d-2)^{2}}\right) \\
& +\eta^{\mu \nu}\left[-\frac{2\left(k \cdot v_{\beta}\right)^{2}}{d-2}+\frac{2 l_{\alpha}^{2}}{(d-2)^{2}}-\frac{2 l_{\beta}^{2}}{(d-2)^{2}}+\frac{4 l_{\alpha}^{2}}{(d-2)^{2}}+\frac{2 l_{\alpha} \cdot l_{\beta}}{(d-2)^{2}}-\left(2 l_{\alpha}^{2}+\frac{3}{2} l_{\alpha} \cdot l_{\beta}\right)\left(\left(v_{\alpha} \cdot v_{\beta}\right)^{2}-\frac{2}{d-2}+\frac{d}{(d-2)^{2}}\right)\right. \\
& \left.+\left(v_{\alpha} \cdot v_{\beta} k \cdot v_{\alpha} k \cdot v_{\beta}+\frac{l_{\alpha} \cdot l_{\beta}}{(d-2)^{2}}\right)\right] .
\end{aligned}
$$

Next, the relation $k^{2}=l_{\alpha}^{2}+2 l_{\alpha} \cdot l_{\beta}+l_{\beta}^{2}=0$ is used to simplify further. The identity $a^{\mu} l_{\beta}^{\nu}=a^{\mu} k^{\nu}-a^{\mu} l_{\alpha}^{\nu}$, and the gauge condition of the gravitational field allows for the gauge-invariant shift $a^{\mu} l_{\beta}^{\nu} \rightarrow \frac{1}{2} a \cdot k \eta^{\mu \nu}-a^{\mu} l_{\alpha}^{\nu}$, since dotting this expression with the polarization tensor would give the same radiation amplitude. Making such changes gives

$$
\begin{aligned}
I^{\mu \nu}= & v_{\alpha}^{\mu} v_{\alpha}^{\nu}\left(2\left(k \cdot v_{\beta}\right)^{2}-\frac{4 l_{\alpha}^{2}}{d-2}\right)+\left(v_{\alpha}^{\mu} v_{\beta}^{\nu}+v_{\alpha}^{\nu} v_{\beta}^{\mu}\right)\left(l_{\alpha}^{2}\left(v_{\alpha} \cdot v_{\beta}\right)-k \cdot v_{\alpha} k \cdot v_{\beta}\right)-2\left(v_{\alpha} \cdot v_{\beta}\right) k \cdot v_{\beta}\left(v_{\alpha}^{\mu} l_{\alpha}^{\nu}+v_{\alpha}^{\nu} l_{\alpha}^{\mu}\right) \\
& +l_{\alpha}^{\mu} l_{\alpha}^{\nu}\left(\left(v_{\alpha} \cdot v_{\beta}\right)^{2}-\frac{1}{d-2}\right)+\eta^{\mu \nu}\left(\frac{2\left(k \cdot v_{\alpha}\right)^{2}}{d-2}+\frac{k \cdot l_{\alpha}}{2}\left(v_{\alpha} \cdot v_{\beta}\right)^{2}-\frac{k \cdot l_{\alpha}}{2(d-2)}\right) \\
& +\eta^{\mu \nu}\left[-\frac{2\left(k \cdot v_{\beta}\right)^{2}}{d-2}+\frac{2 l_{\alpha}^{2}}{(d-2)^{2}}-\frac{2 l_{\beta}^{2}}{(d-2)^{2}}+\frac{4 l_{\alpha}^{2}}{(d-2)^{2}}+\frac{2 l_{\alpha} \cdot l_{\beta}}{(d-2)^{2}}-\left(2 l_{\alpha}^{2}+\frac{3}{2} l_{\alpha} \cdot l_{\beta}\right)\left(\left(v_{\alpha} \cdot v_{\beta}\right)^{2}-\frac{2}{d-2}+\frac{d}{(d-2)^{2}}\right)\right. \\
& \left.+\left(v_{\alpha} \cdot v_{\beta} k \cdot v_{\alpha} k \cdot v_{\beta}+\frac{l_{\alpha} \cdot l_{\beta}}{(d-2)^{2}}\right)\right] .
\end{aligned}
$$

By considering that $\alpha$ and $\beta$ are symmetric, all particle labels may be switched for any term, which allows further simplification to give the final result

$$
\begin{aligned}
I^{\mu \nu}= & v_{\alpha}^{\mu} v_{\alpha}^{\nu}\left(2\left(k \cdot v_{\beta}\right)^{2}-\frac{4 l_{\alpha}^{2}}{d-2}\right)+\left(v_{\alpha}^{\mu} v_{\beta}^{\nu}+v_{\alpha}^{\nu} v_{\beta}^{\mu}\right)\left(l_{\alpha}^{2}\left(v_{\alpha} \cdot v_{\beta}\right)-k \cdot v_{\alpha} k \cdot v_{\beta}\right)-2\left(v_{\alpha} \cdot v_{\beta}\right) k \cdot v_{\beta}\left(v_{\alpha}^{\mu} l_{\alpha}^{\nu}+v_{\alpha}^{\nu} l_{\alpha}^{\mu}\right) \\
& +l_{\alpha}^{\mu} l_{\alpha}^{\nu}\left(\left(v_{\alpha} \cdot v_{\beta}\right)^{2}-\frac{1}{d-2}\right)+\eta^{\mu \nu}\left(v_{\alpha} \cdot v_{\beta} k \cdot v_{\alpha} k \cdot v_{\beta}-\frac{l_{\alpha}^{2}}{2}\left(\left(v_{\alpha} \cdot v_{\beta}\right)^{2}-\frac{1}{d-2}\right)\right) .
\end{aligned}
$$

To more easily compare with the diagrammatic method, $\hat{t}^{\mu \nu}$ is found by adding the lowest-order term of $(1-\sqrt{|g|}) T^{\mu \nu}$, where 


$$
\begin{aligned}
T^{\mu \nu}(x) & \approx \sum_{\alpha=1}^{N} m_{\alpha} \int_{l_{\alpha}}(2 \pi) \delta\left(v_{\alpha} \cdot l_{\alpha}\right) e^{-i l_{\alpha} \cdot\left(x-b_{\alpha}\right)} v_{\alpha}^{\mu} v_{\alpha}^{\nu}, \\
h(x) & \approx \frac{-\kappa}{d-2} \sum_{\beta \neq \alpha} m_{\beta} \int_{l_{\beta}}(2 \pi) \delta\left(l_{\beta} \cdot v_{\beta}\right) \frac{e^{-i l_{\beta} \cdot\left(x-b_{\beta}\right)}}{l_{\beta}^{2}}, \\
(1-\sqrt{|g|}) T^{\mu \nu} & \approx \frac{1}{d-2}\left(\frac{\kappa}{2}\right)^{2} \sum m_{\alpha} m_{\beta} \int_{l_{\alpha}, l_{\beta}} \mu_{\alpha, \beta}(k) 2 l_{\alpha}^{2} v_{\alpha}^{\mu} v_{\alpha}^{\nu} .
\end{aligned}
$$

Adding this to $t^{\mu \nu}$ gives $\hat{t}^{\mu \nu} \equiv\left(\frac{\kappa}{2}\right)^{2} \sum_{\substack{\alpha=1 \\ \beta \neq \alpha}}^{N} m_{\alpha} m_{\beta} \int_{l_{\alpha}, l_{\beta}} \mu_{\alpha, \beta}(k) \hat{I}^{\mu \nu}$, such that

$$
\begin{aligned}
\hat{I}^{\mu \nu}= & v_{\alpha}^{\mu} v_{\alpha}^{\nu}\left(2\left(k \cdot v_{\beta}\right)^{2}-\frac{2 l_{\alpha}^{2}}{d-2}\right)+\left(v_{\alpha}^{\mu} v_{\beta}^{\nu}+v_{\alpha}^{\nu} v_{\beta}^{\mu}\right)\left(l_{\alpha}^{2}\left(v_{\alpha} \cdot v_{\beta}\right)-k \cdot v_{\alpha} k \cdot v_{\beta}\right)-2\left(v_{\alpha} \cdot v_{\beta}\right) k \cdot v_{\beta}\left(v_{\alpha}^{\mu} l_{\alpha}^{\nu}+v_{\alpha}^{\nu} l_{\alpha}^{\mu}\right) \\
& +l_{\alpha}^{\mu} l_{\alpha}^{\nu}\left(\left(v_{\alpha} \cdot v_{\beta}\right)^{2}-\frac{1}{d-2}\right)+\eta^{\mu \nu}\left(v_{\alpha} \cdot v_{\beta} k \cdot v_{\alpha} k \cdot v_{\beta}-\frac{l_{\alpha}^{2}}{2}\left(\left(v_{\alpha} \cdot v_{\beta}\right)^{2}-\frac{1}{d-2}\right)\right) .
\end{aligned}
$$

As shown in the next appendix, this result agrees precisely with a diagram involving the three-point graviton vertex.

\section{APPENDIX B: SOME RADIATIVE FEYNMAN RULES}

\section{Yang-Mills and biadjoint-scalar theory}

A Feynman diagram approach can be used to find the results for diagrams 1(c) and 1(f), shown in Fig. 1. Expanding the kinetic term of the Lagrangian, the $\mathcal{O}\left(A^{3}\right)$ term corresponding to the three-point vector boson interaction is

$$
-\frac{1}{4} F_{\mu \nu}^{a} F^{\mu \nu a}=-\partial_{\mu} A_{\nu}^{a} g f^{a b c} A^{\mu b} A^{\nu c}+\cdots .
$$

This term in the Lagrangian gives the textbook non-Abelian three-point vector boson vertex, given by

$$
\begin{aligned}
\Gamma^{\mu a, \nu b, \rho c}(k, p, q)= & f^{a b c}\left(\left(k^{\nu}-q^{\nu}\right) \eta^{\mu \rho}+\left(p^{\rho}-k^{\rho}\right) \eta^{\nu \mu}\right. \\
& \left.+\left(q^{\mu}-p^{\mu}\right) \eta^{\rho \nu}\right)
\end{aligned}
$$

where $A_{\mu}^{a}$ is associated with the momentum $k, A_{\nu}^{b}$ is associated with $p$, and $A_{\rho}^{c}$ is associated with $q$.

The three-point vertex for two biadjoint scalars and one adjoint vector field can be used to efficiently calculate a piece radiation, which comes from the kinetic term of the biadjoint scalar. Focusing on the terms in the Lagrangian to $\mathcal{O}\left(\Phi^{2} A\right)$

$$
\begin{aligned}
& \frac{1}{2}\left(D_{\mu} \Phi^{\tilde{a}}\right)^{a}\left(D^{\mu} \Phi^{\tilde{b}}\right)^{a} \delta^{\tilde{a} \tilde{b}} \\
& \quad=g f^{a b c} \delta^{\tilde{a} \tilde{c}}\left(\partial_{\mu} \Phi^{\tilde{a} a}\right) A^{\mu b} \Phi^{\tilde{c} c}+\cdots
\end{aligned}
$$

Taking the appropriate functional derivatives and properly symmetrizing gives the three-point vertex for two scalars and one vector,

$$
\Gamma^{\tilde{a} a, \nu b, \tilde{c} c}(k, p, q)=f^{a b c} \delta^{\tilde{a} \tilde{c}}\left(k^{\nu}-q^{\nu}\right)
$$

The three-point vertices above can be used to find diagrams 1(c) and 1(f), giving

$$
\begin{aligned}
(1 \mathrm{c})^{\mu a}(k)= & \left.\frac{1}{2} \int_{l_{\alpha}, l_{\beta}} A_{\nu}^{b}\left(l_{\alpha}\right)\right|_{\mathcal{O}\left(g^{1}\right)} i \Gamma^{\mu a, \nu b, \rho c}\left(-k, l_{\alpha}, l_{\beta}\right) \\
& \times\left. A_{\rho}^{c}\left(l_{\beta}\right)\right|_{\mathcal{O}\left(g^{1}\right)}(2 \pi)^{d} \delta^{d}\left(k-l_{\alpha}-l_{\beta}\right), \\
(1 \mathrm{f})^{\mu a}(k)= & \left.\frac{1}{2} \int_{l_{\alpha}, l_{\beta}} \Phi^{\tilde{b} b}\left(l_{\alpha}\right)\right|_{\mathcal{O}\left(y^{1}\right)} i \Gamma^{\tilde{b} b, \mu a, \tilde{c} c}\left(l_{\alpha},-k, l_{\beta}\right) \\
& \times\left.\Phi^{\tilde{c} c}\left(l_{\beta}\right)\right|_{\mathcal{O}\left(y^{1}\right)}(2 \pi)^{d} \delta^{d}\left(k-l_{\alpha}-l_{\beta}\right),
\end{aligned}
$$

where a symmetry factor of $1 / 2$ has been added.

The solutions needed for these diagrams were found in Eq. (2.16), giving

$$
\begin{aligned}
& \left.A^{\mu a}\left(l_{\alpha}\right)\right|_{\mathcal{O}\left(g^{1}\right)}=-g \sum_{\alpha=1}^{N}(2 \pi) \delta\left(l_{\alpha} \cdot v_{\alpha}\right) \frac{e^{i l_{\alpha} \cdot b_{\alpha}}}{l_{\alpha}^{2}} v_{\alpha}^{\mu} c_{\alpha}^{a}, \\
& \left.\Phi^{a \tilde{a}}\left(l_{\alpha}\right)\right|_{\mathcal{O}\left(y^{1}\right)}=-y \sum_{\alpha=1}^{N}(2 \pi) \delta\left(l_{\alpha} \cdot v_{\alpha}\right) \frac{e^{i l_{\alpha} \cdot b_{\alpha}}}{l_{\alpha}^{2}} c_{\alpha}^{a} c_{\alpha}^{\tilde{a}} .
\end{aligned}
$$

Plugging in these solutions gives

$$
\begin{aligned}
(1 \mathrm{c})^{\mu a}(k)= & \frac{g^{2}}{2} \sum_{\alpha \neq \beta} i f^{a b c} c_{\alpha}^{b} c_{\beta}^{c} \int_{l_{\alpha}, l_{\beta}} \mu_{\alpha, \beta}(k) \\
& \times\left[-2 k \cdot v_{\alpha} v_{\beta}^{\mu}+2 k \cdot v_{\beta} v_{\alpha}^{\mu}+v_{\alpha} \cdot v_{\beta}\left(l_{\beta}-l_{\alpha}\right)^{\mu}\right], \\
(1 \mathrm{f})^{\mu a}(k)= & \frac{y^{2}}{2} \sum_{\beta \neq \alpha} i f^{a b c} c_{\alpha}^{b} c_{\beta}^{c} c_{\alpha}^{\tilde{a}} c_{\beta}^{\tilde{a}} \int_{l_{\alpha}, l_{\beta}} \mu_{\alpha, \beta}(k)\left(l_{\alpha}-l_{\beta}\right)^{\mu} .
\end{aligned}
$$


Because of the antisymmetry of $f^{a b c} c_{\alpha}^{b} c_{\beta}^{c}$, switching $\alpha \leftrightarrow \beta$ for a term multiplied by this factor introduces a minus sign, allowing further simplification,

$$
\begin{aligned}
(1 \mathrm{c})^{\mu a}(k)= & g^{2} \sum_{\alpha \neq \beta} i f^{a b c} c_{\alpha}^{b} c_{\beta}^{c} \int_{l_{\alpha}, l_{\beta}} \mu_{\alpha, \beta}(k) \\
& \times\left[2 k \cdot v_{\beta} v_{\alpha}^{\mu}-\left(v_{\alpha} \cdot v_{\beta}\right) l_{\alpha}^{\mu}\right], \\
(1 \mathrm{f})^{\mu a}(k)= & y^{2} \sum_{\beta \neq \alpha} i f^{a b c} c_{\alpha}^{b} c_{\beta}^{c} c_{\alpha}^{\tilde{a}} c_{\beta}^{\tilde{a}} \int_{l_{\alpha}, l_{\beta}} \mu_{\alpha, \beta}(k) l_{\alpha}^{\mu} .
\end{aligned}
$$

Note how this result agrees with the algebraic method found in Eq. (2.26).

\section{General relativity and Einstein-Yang-Mills theory}

Next, the three-point graviton vertex will be used to stitch together lower order gravitational field solutions to generate a piece of the gravitational radiation field. The three-point graviton vertex from DeWitt [59] and utilized by Sannan [60] is

$$
\begin{aligned}
V_{\mu \alpha, \nu \beta, \sigma \gamma}\left(k_{1}, k_{2}, k_{3}\right)= & \operatorname{sym}\left[-\frac{1}{2} P_{3}\left(k_{1} \cdot k_{2} \eta_{\mu \alpha} \eta_{\nu \beta} \eta_{\sigma \gamma}\right)-\frac{1}{2} P_{6}\left(k_{1 \nu} k_{1 \beta} \eta_{\mu \alpha} \eta_{\sigma \gamma}\right)+\frac{1}{2} P_{3}\left(k_{1} \cdot k_{2} \eta_{\mu \nu} \eta_{\alpha \beta} \eta_{\sigma \gamma}\right)+P_{6}\left(k_{1} \cdot k_{2} \eta_{\mu \alpha} \eta_{\nu \sigma} \eta_{\beta \gamma}\right)\right. \\
& +2 P_{3}\left(k_{1 \nu} k_{1 \gamma} \eta_{\mu \alpha} \eta_{\beta \sigma}\right)-P_{3}\left(k_{1 \beta} k_{2 \mu} \eta_{\alpha \nu} \eta_{\sigma \gamma}\right)+P_{3}\left(k_{1 \sigma} k_{2 \gamma} \eta_{\mu \nu} \eta_{\alpha \beta}\right)+P_{6}\left(k_{1 \sigma} k_{1 \gamma} \eta_{\mu \nu} \eta_{\alpha \beta}\right) \\
& \left.+2 P_{6}\left(k_{1 \nu} k_{2 \gamma} \eta_{\beta \mu} \eta_{\alpha \sigma}\right)+2 P_{3}\left(k_{1 \nu} k_{2 \mu} \eta_{\beta \sigma} \eta_{\gamma \alpha}\right)-2 P_{3}\left(k_{1} \cdot k_{2} \eta_{\alpha \nu} \eta_{\beta \sigma} \eta_{\gamma \mu}\right)\right]
\end{aligned}
$$

where $P_{3}$ and $P_{6}$ refers to a permutation of $k_{1}, k_{2}$, and $k_{3}$ resulting in three or six terms, respectively, and sym applies a symmetrization across $\mu \alpha, \nu \beta$, and $\sigma \gamma$. For example,

$$
\begin{aligned}
P_{3}\left(k_{1} \cdot k_{2} \eta_{\mu \nu} \eta_{\alpha \beta} \eta_{\sigma \gamma}\right) & =k_{1} \cdot k_{2} \eta_{\mu \nu} \eta_{\alpha \beta} \eta_{\sigma \gamma}+k_{2} \cdot k_{3} \eta_{\nu \sigma} \eta_{\beta \gamma} \eta_{\mu \alpha}+k_{3} \cdot k_{1} \eta_{\mu \sigma} \eta_{\alpha \gamma} \eta_{\nu \beta}, \\
\operatorname{sym}\left[\eta_{\mu \nu} \eta_{\alpha \beta}\right] & =\frac{1}{4}\left(\eta_{\mu \nu} \eta_{\alpha \beta}+\eta_{\mu \beta} \eta_{\nu \alpha}+\eta_{\nu \alpha} \eta_{\mu \beta}+\eta_{\alpha \beta} \eta_{\mu \nu}\right) .
\end{aligned}
$$

Expanding $P_{3}$ and $P_{6}$ gives

$$
\begin{aligned}
V^{\mu \alpha, \nu \beta, \sigma \gamma}\left(k_{1}, k_{2}, k_{3}\right)= & \operatorname{sym}\left[-\frac{1}{2}\left(k_{1} \cdot k_{2}+k_{2} \cdot k_{3}+k_{3} \cdot k_{1}\right) \eta^{\mu \alpha} \eta^{\nu \beta} \eta^{\sigma \gamma}\right. \\
& -\frac{1}{2}\left(k_{1}^{\nu} k_{1}^{\beta} \eta^{\mu \alpha} \eta^{\sigma \gamma}+k_{1}^{\sigma} k_{1}^{\gamma} \eta^{\mu \alpha} \eta^{\nu \beta} k_{2}^{\mu} k_{2}^{\alpha} \eta^{\nu \beta} \eta^{\sigma \gamma}+k_{2}^{\sigma} k_{2}^{\gamma} \eta^{\mu \alpha} \eta^{\nu \beta}+k_{3}^{\mu} k_{3}^{\alpha} \eta^{\nu \beta} \eta^{\gamma \sigma}+k_{3}^{\nu} k_{3}^{\beta} \eta^{\mu \alpha} \eta^{\gamma \sigma}\right) \\
& +\frac{1}{2}\left(k_{1} \cdot k_{2} \eta^{\mu \nu} \eta^{\alpha \beta} \eta^{\sigma \gamma}+k_{2} \cdot k_{3} \eta^{\nu \sigma} \eta^{\beta \gamma} \eta^{\mu \alpha}+k_{3} \cdot k_{1} \eta^{\mu \sigma} \eta^{\alpha \gamma} \eta^{\nu \beta}\right) \\
& +\left(k_{1} \cdot k_{2} \eta^{\mu \alpha} \eta^{\nu \sigma} \eta^{\beta \gamma}+k_{1} \cdot k_{2} \eta^{\nu \beta} \eta^{\mu \sigma} \eta^{\alpha \gamma}+k_{2} \cdot k_{3} \eta^{\nu \beta} \eta^{\mu \sigma} \eta^{\alpha \gamma}+k_{2} \cdot k_{3} \eta^{\sigma \gamma} \eta^{\mu \nu} \eta^{\alpha \beta}+k_{3} \cdot k_{1} \eta^{\sigma \gamma} \eta^{\mu \nu} \eta^{\alpha \beta}\right. \\
& \left.+k_{3} \cdot k_{1} \eta^{\mu \alpha} \eta^{\nu \sigma} \eta^{\beta \gamma}\right)+2\left(k_{1}^{\nu} k_{1}^{\gamma} \eta^{\mu \alpha} \eta^{\beta \sigma}+k_{2}^{\sigma} k_{2}^{\mu} \eta^{\nu \beta} \eta^{\gamma \mu}+k_{3}^{\mu} k_{3}^{\beta} \eta^{\sigma \gamma} \eta^{\alpha \nu}\right) \\
& -\left(k_{1}^{\beta} k_{2}^{\mu} \eta^{\alpha \nu} \eta^{\sigma \gamma}+k_{2}^{\gamma} k_{3}^{\nu} \eta^{\beta \sigma} \eta^{\mu \alpha}+k_{3}^{\beta} k_{1}^{\mu} \eta^{\gamma \mu} \eta^{\nu \beta}\right)+\left(k_{1}^{\sigma} k_{2}^{\gamma} \eta^{\mu \nu} \eta^{\alpha \beta}+k_{2}^{\mu} k_{3}^{\alpha} \eta^{\nu \sigma} \eta^{\beta \gamma}+k_{3}^{\nu} k_{1}^{\beta} \eta^{\sigma \mu} \eta^{\gamma \alpha}\right) \\
& +\left(k_{1}^{\sigma} k_{1}^{\gamma} \eta^{\mu \nu} \eta^{\alpha \beta}+k_{1}^{\nu} k_{1}^{\beta} \eta^{\mu \sigma} \eta^{\alpha \gamma}+k_{2}^{\mu} k_{2}^{\alpha} \eta^{\nu \sigma} \eta^{\beta \gamma}+k_{2}^{\sigma} k_{2}^{\gamma} \eta^{\nu \mu} \eta^{\gamma \alpha}+k_{3}^{\nu} k_{3}^{\beta} \eta^{\sigma \mu} \eta^{\gamma \alpha}+k_{3}^{\mu} k_{3}^{\alpha} \eta^{\sigma \nu} \eta^{\alpha \beta}\right) \\
& +2\left(k_{1}^{\nu} k_{2}^{\gamma} \eta^{\beta \mu} \eta^{\alpha \sigma}+k_{1}^{\mu} k_{2}^{\gamma} \eta^{\alpha \nu} \eta^{\beta \sigma}+k_{2}^{\sigma} k_{3}^{\alpha} \eta^{\gamma \nu} \eta^{\beta \mu}+k_{2}^{\nu} k_{3}^{\alpha} \eta^{\beta \sigma} \eta^{\gamma \mu}+k_{3}^{\mu} k_{1}^{\beta} \eta^{\alpha \sigma} \eta^{\gamma \nu}+k_{3}^{\sigma} k_{1}^{\beta} \eta^{\gamma \mu} \eta^{\alpha \sigma}\right) \\
& +2\left(k_{1}^{\nu} k_{2}^{\mu} \eta^{\beta \sigma} \eta^{\gamma \alpha}+k_{2}^{\sigma} k_{3}^{\nu} \eta^{\gamma \mu} \eta^{\alpha \beta}+k_{3}^{\mu} k_{1}^{\sigma} \eta^{\alpha \nu} \eta^{\beta \gamma}\right)-2\left(k_{1} \cdot k_{2} \eta^{\alpha \nu} \eta^{\beta \sigma} \eta^{\gamma \mu}+k_{2} \cdot k_{3} \eta^{\beta \sigma} \eta^{\gamma \mu} \eta^{\alpha \nu}\right. \\
& \left.\left.+k_{3} \cdot k_{1} \eta^{\gamma \mu} \eta^{\alpha \nu} \eta^{\gamma \sigma}\right)\right] .
\end{aligned}
$$

To find the radiative field contribution from this three-point vertex, two instances of the lowest-order field solution will be stitched together with this vertex to find a higher order contribution. The lowest-order field in momentum space is given by

$$
h^{\rho \sigma}\left(l_{\alpha}\right)=\frac{\kappa}{2} \sum_{\alpha}^{N} m_{\alpha} \frac{e^{i l_{\alpha} \cdot b_{\alpha}}}{l_{\alpha}^{2}}(2 \pi) \delta\left(l_{\alpha} \cdot v_{\alpha}\right)\left[v_{\alpha}^{\rho} v_{\alpha}^{\sigma}-\frac{\eta^{\rho \sigma}}{d-2}\right] .
$$


The three-point vertex allows for a purely gravitational source to be found, which corresponds to a component of the pseudotensor, $t^{\mu \nu}$. This component of the source that generates radiation is given by

$$
t^{\sigma \lambda}(k)=\frac{1}{2} \int_{l_{\alpha}, l_{\beta}} V^{\mu \rho, \nu \tau, \sigma \lambda}\left(-l_{\alpha},-l_{\beta}, k\right) h_{\mu \rho}\left(l_{\alpha}\right) h_{\nu \tau}\left(l_{\beta}\right) \delta^{d}\left(k-l_{\alpha}-l_{\beta}\right) .
$$

Since the lowest-order solutions for $l_{\alpha}$ and $l_{\beta}$ are symmetric, the symmetrization is only needed for indices $\sigma$ and $\lambda$ in $V^{\mu \rho, \nu \tau, \sigma \lambda}$. Focusing on the integrand and breaking down the two lowest-order solutions into four terms gives

$$
h^{\mu \rho}\left(l_{\alpha}\right) h^{\nu \tau}\left(l_{\beta}\right) \propto\left[v_{\alpha}^{\mu} v_{\alpha}^{\rho} v_{\beta}^{\nu} v_{\beta}^{\tau}-\frac{1}{d-2}\left(v_{\alpha}^{\mu} v_{\alpha}^{\rho} \eta^{\nu \tau}+v_{\beta}^{\nu} v_{\beta}^{\tau} \eta^{\mu \rho}\right)+\frac{1}{(d-2)^{2}}\left(\eta^{\mu \rho} \eta^{\nu \tau}\right)\right]
$$

Using Mathematica to perform the index contractions gives

$$
\begin{aligned}
(2 c)^{\sigma \tau}(k) & =\left(\frac{\kappa}{2}\right)^{2} \sum_{\substack{\alpha=1 \\
\beta \neq \alpha}}^{N} m_{\alpha} m_{\beta} \int_{l_{\alpha}, l_{\beta}} \mu_{\alpha, \beta}(k)\left[2 v_{\alpha}^{\sigma} v_{\alpha}^{\lambda}\left(\left(k \cdot v_{\beta}\right)^{2}-\frac{l_{\alpha}^{2}}{d-2}\right)+\left(v_{\alpha}^{\sigma} v_{\beta}^{\lambda}+v_{\alpha}^{\lambda} v_{\beta}^{\sigma}\right)\left(l_{\alpha}^{2} v_{\alpha} \cdot v_{\beta}-k \cdot v_{\alpha} k \cdot v_{\beta}\right)\right. \\
& \left.-2\left(v_{\alpha}^{\sigma} l_{\alpha}^{\lambda}+v_{\alpha}^{\lambda} l_{\alpha}^{\sigma}\right)\left(v_{\alpha} \cdot v_{\beta} k \cdot v_{\beta}\right)+l_{\alpha}^{\sigma} l_{\alpha}^{\lambda}\left(\left(v_{\alpha} \cdot v_{\beta}\right)^{2}-\frac{1}{d-2}\right)+\eta^{\sigma \lambda}\left(k \cdot v_{\alpha} k \cdot v_{\beta} v_{\alpha} \cdot v_{\beta}-\frac{l_{\alpha}^{2}}{2}\left(\left(v_{\alpha} \cdot v_{\beta}\right)^{2}-\frac{1}{d-2}\right)\right)\right],
\end{aligned}
$$

where this result gives the integrand of diagram 2(c), as shown in Eq. (3.26).

For calculating the additional gravitational radiation diagrams due to Yang-Mills contributions, the Feynman rules for scattering outlined by Rodigast's thesis give the necessary three-point vertex [61,62]. The Feynman rule for the three-point vertex with two gluons and one graviton can be found from the interaction term in the Lagrangian,

$$
\begin{aligned}
\mathcal{L}= & \sqrt{-g} g^{\mu \rho} g^{\nu \sigma} \partial_{\mu} A_{\nu}^{a} \partial_{[\rho} A_{\sigma]}^{a}+\cdots \\
\approx & \kappa\left(\eta^{\mu \tau} \eta^{\rho \lambda} \eta^{\nu \sigma}+\eta^{\mu \rho} \eta^{\nu \tau} \eta^{\sigma \lambda}-\frac{1}{2} \eta^{\tau \lambda} \eta^{\mu \rho} \eta^{\nu \sigma}\right) \\
& \times h_{\tau \lambda} \partial_{\mu} A_{\nu}^{a} \partial_{[\rho} A_{\sigma]}^{a} .
\end{aligned}
$$

Taking the functional derivatives, and properly symmetrizing over all indices and momenta, gives

$$
\begin{aligned}
\Gamma^{\tau \lambda, \mu \tilde{a}, \nu \tilde{b}}(k, p, q) \\
=-2 i \delta^{\tilde{a} \tilde{b}}\left(p^{(\tau} q^{\lambda)} \eta^{\mu \nu}+\frac{1}{2} p \cdot q\left(\eta^{\tau \mu} \eta^{\lambda \nu} \eta^{\tau \nu} \eta^{\lambda \mu}-\eta^{\tau \lambda} \eta^{\mu \nu}\right)\right. \\
\left.+\frac{1}{2} \eta^{\tau \lambda} p^{\nu} q^{\mu}-q^{\mu} \eta^{\nu(\lambda} p^{\tau)}-p^{\nu} \eta^{\mu(\tau} q^{\lambda)}\right)
\end{aligned}
$$

where a factor of $2 / \kappa$ was added to have the same conventions as DeWitt's three-point vertex. This allows us to use the same formula for calculating the contribution to the radiation source. By reusing the lowest-order result for $\left.A^{\mu a}(l)\right|_{\mathcal{O}\left(g^{1}\right)}$ and switching $a \rightarrow \tilde{a}$, the solution to diagram 2(i) is

$$
\begin{aligned}
(2 \mathrm{i})^{\mu \nu}(k)= & \left.\frac{1}{2} \int_{l_{\alpha}, l_{\beta}} i \Gamma^{\mu \nu, \rho \tilde{a}, \sigma \tilde{b}}\left(-k, l_{\alpha}, l_{\beta}\right) A_{\rho}^{\tilde{a}}\left(l_{\alpha}\right)\right|_{\mathcal{O}\left(g^{1}\right)} \\
& \times\left. A_{\sigma}^{\tilde{b}}\left(l_{\beta}\right)\right|_{\mathcal{O}\left(g^{1}\right)} \delta^{d}\left(k-l_{\alpha}-l_{\beta}\right) .
\end{aligned}
$$

Plugging in the lowest-order solution gives

$$
\begin{aligned}
(2 \mathrm{i})^{\mu \nu}(k)= & \tilde{g}^{2} \sum_{\substack{\alpha=1 \\
\beta \neq \alpha}}^{N} \int_{l_{\alpha}, l_{\beta}} \mu_{\alpha, \beta}(k) c_{\alpha}^{\tilde{a}} c_{\beta}^{\tilde{a}}\left[\frac { 1 } { 2 } \left(v_{\alpha}^{\mu} v_{\beta}^{\nu}\right.\right. \\
& \left.+v_{\alpha}^{\nu} v_{\beta}^{\mu}-\eta^{\mu \nu} v_{\alpha} \cdot v_{\beta}\right) l_{\alpha} \cdot l_{\beta}+v_{\alpha} \cdot v_{\beta} l_{\alpha}^{(\mu} l_{\beta}^{\nu)} \\
& \left.+\frac{1}{2} \eta^{\mu \nu} k \cdot v_{\alpha} k \cdot v_{\beta}-k \cdot v_{\beta} v_{\alpha}^{(\mu} l_{\beta}^{\nu)}-k \cdot v_{\alpha} v_{\beta}^{(\mu} l_{\alpha}^{\nu)}\right],
\end{aligned}
$$

which can be shown to agree with the algebraic result found in Eq. (3.28). 
[1] H. Kawai, D. C. Lewellen, and S. H. H. Tye, A relation between tree amplitudes of closed and open strings, Nucl. Phys. B269, 1 (1986).

[2] Z. Bern, J. J. M. Carrasco, and H. Johansson, New relations for gauge-theory amplitudes, Phys. Rev. D 78, 085011 (2008).

[3] Z. Bern, T. Dennen, Y.-t. Huang, and M. Kiermaier, Gravity as the square of gauge theory, Phys. Rev. D 82, 065003 (2010).

[4] Z. Bern, J. J. M. Carrasco, and H. Johansson, Perturbative Quantum Gravity as a Double Copy of Gauge Theory, Phys. Rev. Lett. 105, 061602 (2010).

[5] N. Arkani-Hamed, F. Cachazo, C. Cheung, and J. Kaplan, A duality for the $S$ matrix, J. High Energy Phys. 03 (2010) 020 .

[6] Y.-X. Chen, Y.-J. Du, and B. Feng, A proof of the explicit minimal-basis expansion of tree amplitudes in gauge field theory, J. High Energy Phys. 02 (2011) 112.

[7] L. de la Cruz, A. Kniss, and S. Weinzierl, Proof of the fundamental BCJ relations for QCD amplitudes, J. High Energy Phys. 09 (2015) 197.

[8] J. J. M. Carrasco and H. Johansson, Generic multiloop methods and application to $N=4$ super-Yang-Mills, J. Phys. A 44, 454004 (2011).

[9] J. J. Carrasco and H. Johansson, Five-point amplitudes in $N=4$ super-Yang-Mills theory and $N=8$ supergravity, Phys. Rev. D 85, 025006 (2012).

[10] Z. Bern, J. J. M. Carrasco, L. J. Dixon, H. Johansson, and R. Roiban, Simplifying multiloop integrands and ultraviolet divergences of gauge theory and gravity amplitudes, Phys. Rev. D 85, 105014 (2012).

[11] Z. Bern, S. Davies, T. Dennen, Y.-t. Huang, and J. Nohle, Color-kinematics duality for pure Yang-Mills and gravity at one and two loops, Phys. Rev. D 92, 045041 (2015).

[12] N. E. J. Bjerrum-Bohr, P. H. Damgaard, and P. Vanhove, Minimal Basis for Gauge Theory Amplitudes, Phys. Rev. Lett. 103, 161602 (2009).

[13] S. Stieberger, Open \& closed vs pure open string disk amplitudes, arXiv:0907.2211.

[14] N. E. J. Bjerrum-Bohr, P. H. Damgaard, T. Sondergaard, and P. Vanhove, Monodromy and Jacobi-like relations for color-ordered amplitudes, J. High Energy Phys. 06 (2010) 003.

[15] S. H. Henry Tye and Y. Zhang, Dual identities inside the gluon and the graviton scattering amplitudes, J. High Energy Phys. 06 (2010) 071; Erratum, 04 (2011) 114(E).

[16] S. He and O. Schlotterer, New Relations for Gauge-Theory and Gravity Amplitudes at Loop Level, Phys. Rev. Lett. 118, 161601 (2017).

[17] S. He, O. Schlotterer, and Y. Zhang, New BCJ representations for one-loop amplitudes in gauge theories and gravity, arXiv:1706.00640.

[18] T. Adamo, E. Casali, K. A. Roehrig, and D. Skinner, On tree amplitudes of supersymmetric Einstein-Yang-Mills theory, J. High Energy Phys. 12 (2015) 177.

[19] L. de la Cruz, A. Kniss, and S. Weinzierl, Relations for Einstein-Yang-Mills amplitudes from the CHY representation, Phys. Lett. B 767, 86 (2017).

[20] S. Stieberger and T. R. Taylor, New relations for EinsteinYang-Mills amplitudes, Nucl. Phys. B913, 151 (2016).
[21] Y.-J. Du, F. Teng, and Y.-S. Wu, Direct evaluation of $n$-point single-trace MHV amplitudes in 4d Einstein-Yang-Mills theory using the CHY formalism, J. High Energy Phys. 09 (2016) 171.

[22] F. Cachazo, S. He, and E. Y. Yuan, Einstein-Yang-Mills scattering amplitudes from scattering equations, J. High Energy Phys. 01 (2015) 121.

[23] D. Nandan, J. Plefka, O. Schlotterer, and C. Wen, EinsteinYang-Mills from pure Yang-Mills amplitudes, J. High Energy Phys. 10 (2016) 070.

[24] O. Schlotterer, Amplitude relations in heterotic string theory and Einstein-Yang-Mills, J. High Energy Phys. 11 (2016) 074.

[25] F. Cachazo, S. He, and E. Y. Yuan, Scattering equations and Kawai-Lewellen-Tye orthogonality, Phys. Rev. D 90, 065001 (2014).

[26] C. D. White, Exact solutions for the biadjoint scalar field, Phys. Lett. B 763, 365 (2016).

[27] M. Chiodaroli, M. Gnaydin, H. Johansson, and R. Roiban, Scattering amplitudes in $\mathcal{N}=2$ Maxwell-Einstein and Yang-Mills/Einstein supergravity, J. High Energy Phys. 01 (2015) 081.

[28] M. Chiodaroli, M. Gunaydin, H. Johansson, and R. Roiban, Spontaneously broken Yang-Mills-Einstein supergravities as double copies, J. High Energy Phys. 06 (2017) 064.

[29] M. Chiodaroli, Simplifying amplitudes in Maxwell-Einstein and Yang-Mills-Einstein supergravities, arXiv:1607.04129.

[30] B. P. Abbott et al. (LIGO Scientific Collaboration and Virgo Collaboration), Observation of Gravitational Waves from a Binary Black Hole Merger, Phys. Rev. Lett. 116, 061102 (2016).

[31] A. Anastasiou, L. Borsten, M. J. Duff, L. J. Hughes, and S. Nagy, A magic pyramid of supergravities, J. High Energy Phys. 04 (2014) 178.

[32] A. Anastasiou et al., Are all supergravity theories YangMills squared?, arXiv:1707.03234.

[33] H. Johansson and J. Nohle, Conformal gravity from gauge theory, arXiv:1707.02965.

[34] R. Monteiro, D. O'Connell, and C. D. White, Black holes and the double copy, J. High Energy Phys. 12 (2014) 056.

[35] A. K. Ridgway and M. B. Wise, Static spherically symmetric Kerr-Schild metrics and implications for the classical double copy, Phys. Rev. D 94, 044023 (2016).

[36] A. Luna, R. Monteiro, D. O'Connell, and C. D. White, The classical double copy for Taub-NUT spacetime, Phys. Lett. B 750, 272 (2015).

[37] A. Luna, R. Monteiro, I. Nicholson, D. O'Connell, and C.D. White, The double copy: Bremsstrahlung and accelerating black holes, J. High Energy Phys. 06 (2016) 023.

[38] N. Bahjat-Abbas, A. Luna, and C. D. White, The KerrSchild double copy in curved spacetime, J. High Energy Phys. 12 (2017) 004.

[39] T. Adamo, E. Casali, L. Mason, and S. Nekovar, Scattering on plane waves and the double copy, Classical Quantum Gravity 35, 015004 (2018).

[40] A. Luna, I. Nicholson, D. O'Connell, and C. D. White, Inelastic black hole scattering from charged scalar amplitudes, J. High Energy Phys. 03 (2018) 044. 
[41] W. D. Goldberger and A. K. Ridgway, Radiation and the classical double copy for color charges, Phys. Rev. D 95, 125010 (2017).

[42] W. D. Goldberger, S. G. Prabhu, and J. O. Thompson, Classical gluon and graviton radiation from the bi-adjoint scalar double copy, Phys. Rev. D 96, 065009 (2017).

[43] C. Misner, K. Thorne, and J. Wheeler, Gravitation (W. H. Freeman, San Francisco, 1973), https://books.google.com/ books?id=w4Gigq3tY1kC.

[44] Y.-Z. Chu, More on cosmological gravitational waves and their memories, Classical Quantum Gravity 34, 194001 (2017).

[45] C. M. Will and A. G. Wiseman, Gravitational radiation from compact binary systems: Gravitational wave forms and energy loss to second post-Newtonian order, Phys. Rev. D 54, 4813 (1996).

[46] E. E. Flanagan and S. A. Hughes, The basics of gravitational wave theory, New J. Phys. 7, 204 (2005).

[47] L. Blanchet, Gravitational radiation from post-Newtonian sources and inspiralling compact binaries, Living Rev. Relativity 17, 2 (2014).

[48] R. A. Porto and I. Z. Rothstein, Apparent ambiguities in the post-Newtonian expansion for binary systems, Phys. Rev. D 96, 024062 (2017).

[49] L. Bernard, L. Blanchet, G. Faye, and T. Marchand, Centerof-mass equations of motion and conserved integrals of compact binary systems at the fourth post-Newtonian order, Phys. Rev. D 97, 044037 (2018).

[50] A. Boh et al., Improved effective-one-body model of spinning, nonprecessing binary black holes for the era of gravitational-wave astrophysics with advanced detectors, Phys. Rev. D 95, 044028 (2017).

[51] J. Moxon and A. Flanagan, Radiation-reaction force on a small charged body to second order, arXiv:1711.05212.
[52] T. Damour, Gravitational scattering, post-Minkowskian approximation and effective one-body theory, Phys. Rev. D 94, 104015 (2016).

[53] D. Bini and T. Damour, Gravitational spin-orbit coupling in binary systems, post-Minkowskian approximation and effective one-body theory, Phys. Rev. D 96, 104038 (2017).

[54] T. Damour, High-energy gravitational scattering and the general relativistic two-body problem, Phys. Rev. D 97, 044038 (2018).

[55] P. Sikivie and N. Weiss, Classical Yang-Mills theory in the presence of external sources, Phys. Rev. D 18, 3809 (1978).

[56] J. D. Jackson, Classical Electrodynamics 3rd ed. (Wiley, New York, 1999), http://cdsweb.cern.ch/record/490457.

[57] P. Dirac, General Theory of Relativity, Physics Notes (Princeton University Press, Princeton, NJ, 1975), https:// books.google.com/books?id=qkWPDAAAQBAJ.

[58] L. Landau and E. Lifshitz, in The Classical Theory of Fields (Fourth Edition), edited by L. Landau and E. Lifshitz, Course of Theoretical Physics Vol. 2, (Pergamon, Amsterdam, 1975), Chap. 11, pp. 259-294, https://www.sciencedirect .com/science/article/pii/B9780080250724500186.

[59] B. S. DeWitt, Quantum theory of gravity. III. Applications of the covariant theory, Phys. Rev. 162, 1239 (1967).

[60] S. Sannan, Gravity as the limit of the type-ii superstring theory, Phys. Rev. D 34, 1749 (1986).

[61] D. Ebert, J. Plefka, and A. Rodigast, Absence of gravitational contributions to the running Yang-Mills coupling, Phys. Lett. B 660, 579 (2008).

[62] A. Rodigast, Ph. D. thesis, Humboldt University, 2008, http://people.physik.hu-berlin.de/sylvia/qftpha/qft/ downloads/DA-andreas.pdf. 